\title{
Fitting and forecasting coupled dark energy in the non-linear regime
}

\author{
Santiago Casas ${ }^{1}$, Luca Amendola ${ }^{1}$, Marco Baldi ${ }^{2,3,4}$, Valeria Pettorino ${ }^{1}$, Adrian Vollmer ${ }^{1}$ \\ ${ }^{1}$ Institut für Theoretische Physik, Ruprecht-Karls-Universität Heidelberg, Philosophenweg 16, 69120 Heidelberg, Germany \\ ${ }^{2}$ Dipartimento di Fisica e Astronomia, Alma Mater Studiorum Università di Bologna, viale Berti Pichat, 6/2, I-40127 Bologna, Italy; \\ ${ }^{3}$ INAF - Osservatorio Astronomico di Bologna, via Ranzani 1, I-40127 Bologna, Italy; \\ ${ }^{4}$ INFN - Sezione di Bologna, viale Berti Pichat 6/2, I-40127 Bologna, Italy.
}

\begin{abstract}
We consider cosmological models in which dark matter feels a fifth force mediated by the dark energy scalar field, also known as coupled dark energy. Our interest resides in estimating forecasts for future surveys like Euclid when we take into account non-linear effects, relying on new fitting functions that reproduce the nonlinear matter power spectrum obtained from $\mathrm{N}$-body simulations.

We obtain fitting functions for models in which the dark matter-dark energy coupling is constant. Their validity is demonstrated for all available simulations in the redshift range $z=0-1.6$ and wave modes below $k=10 \mathrm{~h} / \mathrm{Mpc}$. These fitting formulas can be used to test the predictions of the model in the non-linear regime without the need for additional computing-intensive N-body simulations. We then use these fitting functions to perform forecasts on the constraining power that future galaxy-redshift surveys like Euclid will have on the coupling parameter, using the Fisher matrix method for galaxy clustering (GC) and weak lensing (WL). We find that by using information in the non-linear power spectrum, and combining the GC and WL probes, we can constrain the dark matter-dark energy coupling constant squared, $\beta^{2}$, with precision smaller than $4 \%$ and all other cosmological parameters better than $1 \%$, which is a considerable improvement of more than an order of magnitude compared to corresponding linear power spectrum forecasts with the same survey specifications.
\end{abstract}

\section{INTRODUCTION}

Cosmological data have reached a level of precision that forces us to address effects which may have been safely neglected in the past. Among them, predicting the behaviour of theoretical models at non-linear scales gives a more concrete chance to disentangle $\Lambda \mathrm{CDM}$ from alternative scenarios. The non-linear power spectrum contains useful information encoded in the clustering of galaxies and the weak lensing correlations, which can help to constrain more tightly the cosmological parameters and remove certain degeneracies between them. The drawback relies in the fact that the full estimate of the non-linear power spectrum requires time-demanding and computationally expensive N-body simulations. As a consequence, there exist simulations only for a handful of models beyond $\Lambda \mathrm{CDM}$ on a very limited subset of the parameter space of dark energy theories. This makes it hard to use the non-linear power spectrum in forecasts (see e.g. [1]), both when doing cosmological parameter estimation via Monte Carlo simulations and with the simpler approximation of the Fisher matrix formalism. The alternative is to employ fitting functions for the non-linear power spectrum, like Halofit [2, 3] or machine learning estimators like the cosmological emulator (CosmicEmu) [4-6] and the interpolator PkANN [7, 8] based on artificial neural networks. All these methods are calibrated or trained using large sets of N-body simulations and typically permit an exploration of the parameter space just around a fiducial $\Lambda \mathrm{CDM}$ cosmology or $w \mathrm{CDM}$ cosmology with a constant $w$. An extension of Halofit to account for massive neutrinos was realised in [9], which was then used in [10] to perform a parameter estimation using a Markov Chain Monte Carlo technique. Other authors have recently used polynomial fits to take into account systematic effects in the non-linear regime like baryonic effects such as in [11] or to parametrize the cosmological dependence of non-linear clustering, beyond the Zeldovich approximation (see [12]). These fitting functions have, however, an intrinsic error that usually increases when scales become highly non-linear; therefore one has to be aware of the range in scales and redshifts they were designed to work on, in order to keep the errors induced on the power spectrum under control.

As an alternative to fitting formulae and N-body simulations, there has been in recent years a substantial progress in semianalytical methods to calculate the non-linear power spectrum at mildly non-linear scales, such as for example renormalized perturbation theory [13] and all other resummation methods derived from it (for example [14]), the time renormalisation group (TRG) [15] and effective field theories of large scale structure [16]. Most of these approaches claim to reach percent accuracy at the baryon acoustic oscillation (BAO) scale and a bit beyond the second BAO peak, but they are still not able to predict what happens at highly non-linear scales, when the single stream approximation does not hold any longer.

In this paper, we will use N-Body simulations to find fitting functions for a class of models beyond $\Lambda$ CDM usually refered to as coupled Dark Energy (CDE). These models, widely discussed in literature [17--20], involve an extra degree of freedom, associated to a scalar field that provides acceleration and mediates a fifth force, in addition to gravity, which is felt by dark matter particles only. Semi-analytical non-linear analysis [21, 22] and cosmological N-body simulations within coupled Dark Energy have been performed by many different groups [23-28] and their effects on large scale structure formation have been identified and characterised. The power spectrum, halo mass functions and concentration, halo spin and sphericity, voids and amount of substructures show noticeable differences compared to a simple $\Lambda$ CDM model (see for example [29-34] and the review article [35]). For a constant coupling, constraints have been found for a variety of probes [36-39], with the latest ones discussed by the Planck collaboration in [40]. Recently there have been attempts to constrain more general couplings between dark matter (DM) 
and dark energy (DE) using large-scale structure [41], CMB [42] or laboratory experiments [43]. Forecasts on coupled dark energy using galaxy clustering (GC) and weak lensing (WL) measurements for future surveys like Euclid have been discussed in [44], but have been performed using only linear power spectra for the CDE models. The TRG method has been extended to coupled Dark Energy [45] and to massive neutrinos [46]. However the TRG method does not produce a reliable estimation of the power spectrum for scales larger than $k \approx 0.3 h / \mathrm{Mpc}$, which makes them less suitable for forecasts which attempt to extract information on highly non-linear scales.

The CoDECS (Coupled Dark Energy Cosmological Simulations) set of N-body simulations [27] has shown that CDE models have characteristic and measurable features in the morphology and history of non-linear structures, such as halos, subhalos and voids, and therefore in the non-linear power spectrum.

The aim of this paper is to create fitting functions which are valid in the observable regime of non-linear perturbations at all interesting redshifts and reproduce the subtle effects of coupled dark energy on the non-linear power spectrum while allowing us to vary the different parameters of the model. We use this to perform forecasts of cosmological parameters assuming coupled dark energy as the fiducial model, using galaxy clustering and weak lensing as observational tools, as expected for future surveys like Euclid [47, 48]. We do a careful treatment of errors and systematics, so that we take into account all errors induced by our fitting functions, the cosmic emulators and the extraction of the power spectrum from the N-body simulation into the analysis, systematics related to non-linear effects that affect the redshift space distortions and the lensing signals. In this way we obtain a conservative estimate on how well a probe like Euclid, will be able to measure a DM-DE coupling.

\section{COUPLED DARK ENERGY}

In this section we briefly review the main equations governing the coupled DE models that will be investigated in the present work. For a more detailed and extended discussion and for the full derivation of the equations we refer the interested reader to some of the original publications on the subject [18,-20, 23].

The background evolution for the coupled DE scenario model is described by the following equations, in which the subscripts $r, b, c$ and $\phi$, indicate radiation, baryons, cold dark matter (CDM) and the dark energy scalar field, respectively:

$$
\begin{aligned}
\ddot{\phi}+3 H \dot{\phi}+\frac{d V}{d \phi} & =\sqrt{\frac{2}{3}} \beta(\phi) \frac{\rho_{c}}{M_{P l}}, \\
\dot{\rho}_{c}+3 H \rho_{c} & =-\sqrt{\frac{2}{3}} \beta(\phi) \frac{\rho_{c} \dot{\phi}}{M_{P l}}, \\
\dot{\rho}_{b}+3 H \rho_{b} & =0, \\
\dot{\rho}_{r}+4 H \rho_{r} & =0, \\
3 H^{2} & =\frac{1}{M_{P l}^{2}}\left(\rho_{b}+\rho_{c}+\rho_{r}+\rho_{\phi}\right) .
\end{aligned}
$$

We express from now on the scalar field $\phi$ in units of the Planck mass $M_{p l} \equiv 1 / \sqrt{8 \pi G}$, and choose as potential $V(\phi)$ an exponential $V(\phi)=A e^{-\alpha \phi}$ [49, 50]. The coupling function $\beta(\phi)$ defines the strength of the interaction between the DE fluid and CDM particles and in the present work we will restrict our analysis to the simplified case of a constant coupling $\beta(\phi)=\beta$, although in general it could be a field-dependent quantity [19, 51].

The current constraints on a coupling to ordinary matter are very tight. The "post-Einstein" coupling parameter $\bar{\gamma}$ that measures the local admixture of a spin-0 field to gravity is constrained in Solar System experiments (see e.g. the PDG review [52] and also [53, 54]) roughly to $|\bar{\gamma}| \leq 4 \cdot 10^{-5}$. This parameter enters the modification of the effective Newton constant as $G_{e f f}=G_{N}(1-$ $\bar{\gamma} / 2)$; in our notation, this is $G_{e f f}=G_{N}\left(1+4 \beta^{2} / 3\right.$ ) (see below) and therefore $\beta^{2}=-3 \bar{\gamma} / 8$. A coupling $\beta_{\text {baryons }}^{2}$ appears then constrained to be smaller than $10^{-5}$ roughly, and we assume therefore that is completely negligible. As a consequence, baryons follow the usual geodesics of a FLRW cosmology, which allows coupled DE to pass the stringent local gravity constraints without the need to employ any screening mechanism [55].

Due to the exchange of energy between DE and CDM, the energy density of the latter will no longer scale as the cosmic volume, and by assuming the conservation of the CDM particle number one can derive the time evolution of the CDM particle mass by integrating Eq. (2) between the present time $(z=0)$ and any other redshift $z$ :

$$
m_{c}(z)=m_{c, 0} e^{-\beta(\phi(z)-\phi(0))} .
$$

At the level of linear perturbations, coupled DE models are characterised by a different evolution of the baryonic and CDM density fluctuations, as a consequence of the selective interaction between DE and CDM particles only. In the sub-horizon limit, 


\begin{tabular}{ccc|c} 
Parameter & Explanation & Value & Reference $\Lambda$ CDM \\
\hline \hline$A$ & Potential normalization & 0.0218 & - \\
$\alpha$ & Potential slope & 0.08 & - \\
$\phi(z=0)$ & Scalar field normalization & 0 & - \\
$\beta$ & Coupling parameter & $\{0.05,0.10,0.15\}$ & - \\
$w_{\phi}(z=0)$ & DE equation of state & $\{-0.997,-0.995,-0.992\}$ & -1 \\
$\sigma_{8}(z=0)$ & Power spectrum amplitude & $\{0.825,0.875,0.967\}$ & 0.809 \\
\hline
\end{tabular}

Table I. The main parameters and normalizations of the three coupled DE models of the CoDECS suite considered in the present work. The last column displays the corresponding values for the reference $\Lambda \mathrm{CDM}$ cosmology.

for which $a H / k \ll 1$, linear perturbations in coupled dark energy follow the equations [19, 20]:

$$
\begin{aligned}
& \ddot{\delta}_{c}=-2 H\left[1-\beta \frac{\dot{\phi}}{H \sqrt{6}}\right] \dot{\delta}_{c}+4 \pi G\left[\rho_{b} \delta_{b}+\rho_{c} \delta_{c} \Gamma_{c}\right] \\
& \ddot{\delta}_{b}=-2 H \dot{\delta}_{b}+4 \pi G\left[\rho_{b} \delta_{b}+\rho_{c} \delta_{c}\right]
\end{aligned}
$$

where $\Gamma_{c} \equiv 1+\frac{4}{3} \beta^{2}$ represents the effective "fifth force" acting on the CDM particles. The term proportional to $\beta \dot{\phi}$ in equation 77 is a velocity-dependent term that modifies the standard cosmological friction; this arises as a consequence of momentum conservation for the CDM particles and has a considerable effect on structure formation [23, 24, 31]. Since baryons are uncoupled, their perturbations evolve according to the standard equation. Nonetheless, baryons will still be indirectly affected by the coupling as the source term on the right-hand side of equation (8) includes the CDM density perturbations.

At the level of non-linear perturbations, several methods have been devised to predict the small scale effects of coupled DE, from semi-analytical methods like spherical collapse [56], to time renormalisation group [22] to full N-body simulations [23, 24, 57, 58]. In the following, we will work with the publicly available data of the CoDECS simulations [27] that represents the largest set of cosmological N-body simulations for coupled DE models to date.

\section{THE CODECS SIMULATIONS}

The CoDECS suite ${ }^{1}$ includes simulated periodic volumes of the universe at different scales and with different physical ingredients (as e.g. simulations with and without hydrodynamics) in the context of a series of coupled DE cosmologies characterised by various choices of the self-interaction potential $V(\phi)$ and of the coupling functions $\beta(\phi)$. The simulations have been performed with a suitably modified version (see [27] for more details on the numerical implementation) of the widely-used TreePM N-body code GADGET [59]. Such modified version includes all the relevant effects that characterise coupled DE cosmologies, from the modified background evolution to the CDM particle mass variation, the "fifth-force" and the velocity-dependent acceleration appearing in equation (7).

As already stated above, in this work we will consider - besides the reference $\Lambda$ CDM simulation - the subset of CoDECS runs characterised by an exponential potential and by a constant coupling function. This consists of three different coupled DE models with the same potential slope $\alpha$ and with three values of the coupling $\beta=\{0.05,0,1,0.15\}$. The short names for these simulations are respectively EXP001, EXP002 and EXP003. All the models have been built in order to have the same cosmological parameters at $z=0$ consistent with the WMAP7 results [60], see Table II with the obvious exception of the value of the equation of state parameter $w_{0}$, that changes from model to model due to the different dynamics of the DE scalar field. The present observational constraints on the cosmological parameters have only slightly changed with the latest updated release of Planck data [61] with respect to the assumed WMAP7 values, and are still good enough for the purposes of this paper, being in good agreement with large scale structure observations. For what concerns linear perturbations, all cosmologies have been normalised to have the same statistics (i.e. the same power spectrum shape and amplitude) of density fluctuations at the redshift of the Cosmic Microwave Background $z_{\mathrm{CMB}} \approx 1100$. As a consequence of this choice and of the different growth associated with the various coupling values, all the models will have a different normalisation $\sigma_{8}$ of the linear perturbations amplitude at $z=0$. The main features of these models are summarised in Table II We refer to [27] for further details.

For the purposes of the present work, we will employ the matter power spectra extracted from the CoDECS runs of these cosmologies at different redshifts in order to find a fitting formula that captures with high accuracy the deviations of the coupled $\mathrm{DE}$ nonlinear power spectra from the reference $\Lambda \mathrm{CDM}$ case.

\footnotetext{
${ }^{1}$ see also the public CoDECS database at www.marcobaldi.it/CoDECS/
} 


\begin{tabular}{cc} 
Parameter & Value \\
\hline$H_{0}$ & $70.3 \mathrm{kms}^{-1} \mathrm{Mpc}^{-1}$ \\
$\Omega_{C D M}$ & 0.226 \\
$\Omega_{D E}$ & 0.729 \\
$\mathscr{A}_{s}$ & $2.42 \times 10^{-9}$ \\
$\Omega_{b}$ & 0.0451 \\
$n_{s}$ & 0.966 \\
\hline
\end{tabular}

Table II. The set of cosmological parameters used in all CoDECS simulations, consistent with the WMAP7 results.

\section{EXTRACTING THE POWER SPECTRUM AT SMALL SCALES}

The power spectrum $P(k)$, is defined as the ensemble average of the density contrast in Fourier space $\left\langle\delta(k) \delta\left(k^{\prime}\right)\right\rangle \equiv$ $(2 \pi)^{3} P(k) \delta_{D}\left(k+k^{\prime}\right)$. When trying to extract this quantity from an $\mathrm{N}$-body simulation, one has to take into account several technicalities related to sampling effects which appear as a consequence of treating a discrete distribution of particles. For more details related to this problem and different solution methods, see [62].

At large scales (or equivalently small $k$ ) the power spectrum suffers from uncertainties due to the finite size of the simulation box, since there are only few independent modes to sample the signal from. On the other hand, at high $k$, one is limited by the resolution of the simulation, since one cannot sample wave modes smaller than the typical grid size $L / N$ where $L$ is the length of one side of the simulation box and $N$ is the number of particles. This maximum frequency is called the Nyquist frequency $k_{N y} \equiv 2 \pi N / L$ and modes smaller than this cannot be reliably measured (this corresponds to the so-called aliasing effect). For the CoDECS simulations used in this work, the Nyquist frequency has the value $k_{N y} \approx 2.2 \mathrm{~h} / \mathrm{Mpc}$ at present time.

The power spectrum computation embedded in GADGET-3 that was adopted in the CoDECS simulations employs the so-called folding method developed by [63] - which is based on [64] - to calculate the matter power spectrum for smaller scales than the Nyquist frequency. Following this method one ends up with two separate power spectra, $P_{t o p}$ which is calculated using the simulation particle mesh (PM) at $k \lesssim k_{N y}$ and $P_{\text {fold }}$ which is the folded power valid for $k \gtrsim k_{N y}$. In order to provide a single sampling of the power spectrum across $k_{N y}$ the CoDECS project employed a simple interpolation procedure around $k_{N y}$ by averaging the two power spectra in the region of overlap. However, this might introduce some spurious features that appear only when considering the ratio of two power spectra $P(k)$ at highly non-linear scales. Although such features are harmless for most practical purposes, for the aims of the present work it is very important to have accurate ratios of power spectra, since we want to calculate fitting functions that can capture the effect of the dark energy - dark matter coupling compared to $\Lambda \mathrm{CDM}$ and therefore we need to correct for these spurious effects.

We then developed a PYTHON code that finds the optimal interpolation and matching between the $P_{\text {top }}$ and $P_{\text {fold }}$ power spectra. By evaluating the first and second derivatives of the ratios and minimizing abrupt changes, it finds the optimal number of points to be removed from $P_{\text {top }}$ due to the aliasing error and the number of points to be removed from the $P_{\text {fold }}$ due to the low sampling error; at the same time it looks for the optimal linear interpolation weights between them. Moreover, it cuts off the power spectrum when the shot noise error $\left(P_{\text {shot }}=1 / N\right)$ reaches $10 \%$ of the estimated power spectrum. This method improves considerably the convergence of the fitting functions at non-linear scales, allowing us to reach our accuracy goal of 1-2\% (see section $\mathrm{V}$ on fitting functions). In figure (4), the uncertainties on $P(k)$ are plotted, and the shaded region represents the error on the power spectrum due to the finite number of modes available. The clear jump present in this shaded region occurs at the scale in which the folded and top level power spectra have been matched together, which corresponds roughly to $k_{N y}$.

\section{FITTING FUNCTIONS}

\section{A. The net effect of the DM-DE coupling at non-linear scales}

We now model the net effect of a coupled DE model with an exponential potential and a constant coupling on top of a fiducial $\Lambda \mathrm{CDM}$ non-linear power spectrum, by evaluating the ratio of the non-linear power spectrum of the coupled DE model, with respect to the one in the $\Lambda$ CDM model, both extracted from the CoDECS simulations. In figure $(1)$ we show the ratio $R(k ; \beta, z) \equiv$ $P_{\operatorname{Exp}}(k ; \beta, z) / P_{L C D M}(k ; \beta, z)$ as a function of the scale $\mathrm{k}$, for four different redshifts $z=\{0,0.55,1.0,1.61\}$; each panel contains the curves corresponding to the three available constant couplings in the CoDECS simulations: $\beta=\{0.05,0.10,0.15\}$. Since all coupled DE models have the same amplitude of perturbations at recombination, an increasing coupling has the effect of inducing a higher linear normalization $\sigma_{8}$ of the power spectrum at $z=0$ [27]. Therefore, in order to see the net effect of the coupling at non-linear scales, each power spectrum ratio has been re-normalized by dividing each model by its respective $\sigma_{8}^{2}$, so that at linear scales $k \lesssim 0.1 \mathrm{~h} / \mathrm{Mpc}$ all the ratios are unity. The net effect of the fifth force is a "bump" at non-linear scales, whose amplitude increases with higher couplings and whose maximum is shifted into higher wavenumbers $k$ for higher redshifts. This 

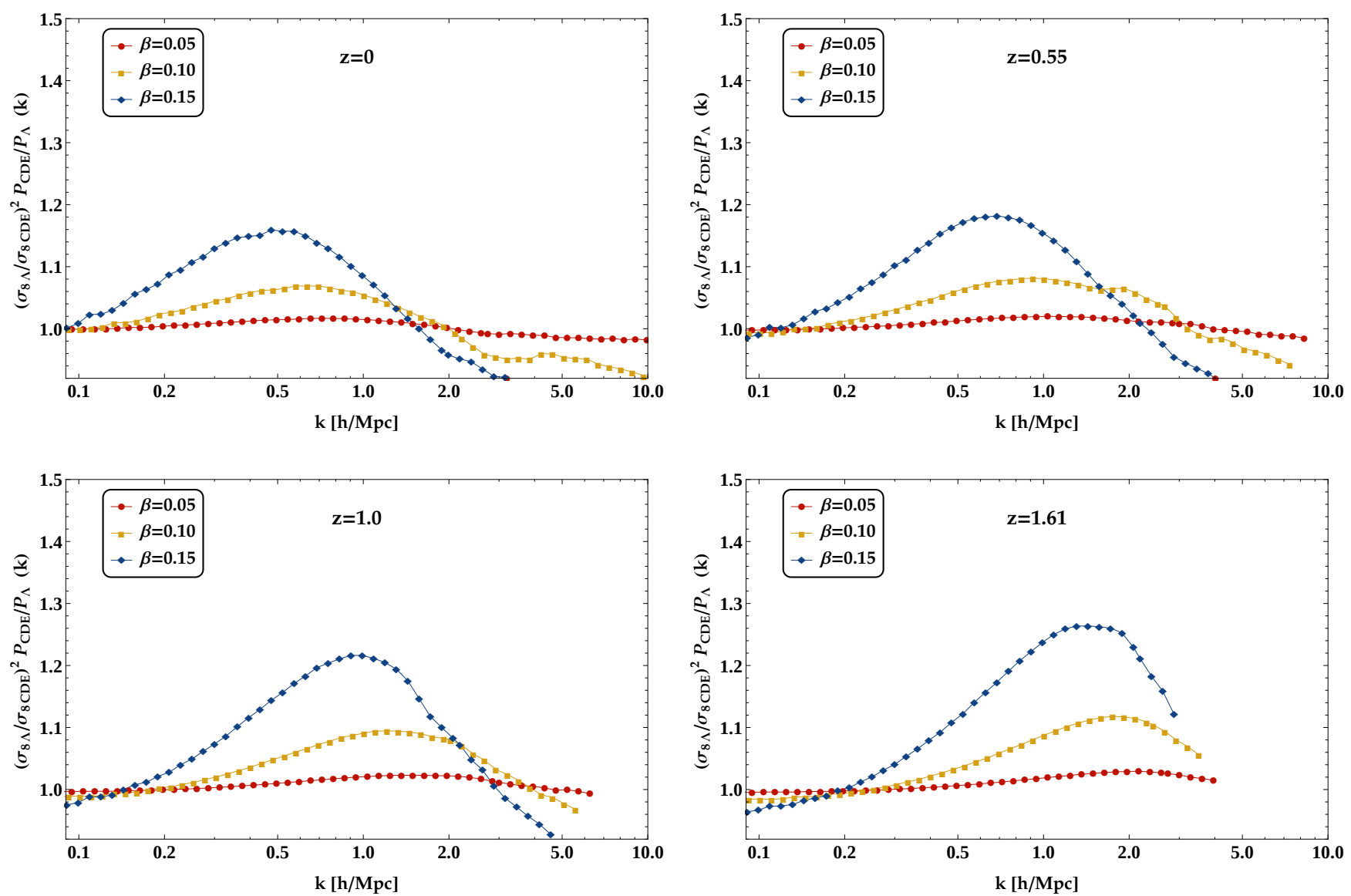

Figure 1. Ratios of the non-linear matter power spectra of the CODECS CDE models with an exponential potential, with respect to the CODECS $\Lambda$ CDM power spectra, normalized by their respective $\sigma_{8}^{2}$, evaluated at four different redshifts and three different coupling constants $\beta$. Upper left panel: $z=0$, upper right panel: $z=0.55$, lower left panel: $z=1.0$, lower right panel: $z=1.61$. The blue line represents the model with strongest coupling $\beta=0.15$, the yellow line $\beta=0.10$ and the red line the smallest available coupling $\beta=0.05$. In order to be able to observe the net effect of the $\beta$ coupling at non-linear scales we have divided each power spectrum by its respective $\sigma_{8}^{2}$.

extra information imprinted into the non-linear power spectrum is what we want to use to improve the estimation of parameters using future surveys. To achieve this, we will fit these curves which are functions of redshift, physical scale and coupling, using the minimal number of numerical parameters possible, while keeping the accuracy goal at the $1 \%$ level.

\section{B. Generating the fitting functions}

The fact that one can observe a clear trend that relates the amplitude of the signal to an increase of the coupling, together with a shift of the peak towards larger length scales when looking at smaller redshifts, is an indication that we should be able to find a relatively simple fitting formula describing this behaviour, which will be then a function of $z, k$ and the coupling constant $\beta$ only.

To perform the fit, we use a least-squares-minimizing technique, using the conjugate gradient method [65]. Taking into account the particular form in $k$-space of the ratios $R(k ; \beta, z) \equiv P_{E X P}(k ; \beta, z) / P_{L C D M}(k ; \beta, z)$ that we need to fit, we use as an Ansatz different sigmoid functions to reproduce the particular form of the peak. For each fitting model, we keep the same functional form for the $k$-dependence of $R(k ; \beta, z)$ at all redshifts and for all couplings. We tried 7 different sigmoid functions as fitting models, but we only show the best two models M2 and M7 in table III All models contain 5 coefficients, which are dependent on the coupling $\beta$ and the redshift $z:\left(a_{i}=a_{0}, a_{1}, c, b, k_{0}\right.$, with $\left.i=0, \ldots, 5\right)$. The coefficients $k_{0}$ and $b$ determine qualitatively the form of the peak to be fitted, while the others control mostly the shifting and the flattening of the peak.

Each coefficient $a_{i}$ is then fitted using a polynomial in $\beta$ and $z$, up to a maximum of third order in powers of $\beta$ and $z$. Polynomials of order 4 and 5 were also examined, but the gain in goodness of fit was minimal compared to the increase in the 


\begin{tabular}{|c|c|c|c|}
\hline Model Name & Functional form & \# of coefficients & $\mathrm{R}^{2}$-value \\
\hline \hline M2 & $f(k)=1+a_{0}+a_{1} \cdot k+c \cdot k \cdot \arctan \left(\left(k-k_{0}\right) \cdot b\right)$ & 5 & 0.99996 \\
\hline M7 & $f(k)=1+a_{0}+a_{1} \cdot k+c \cdot k \cdot \frac{b \cdot\left(k-k_{0}\right)}{\sqrt{1+b^{2} \cdot\left(k-k_{0}\right)^{2}}}$ & 5 & 0.999989 \\
\hline
\end{tabular}

Table III. Fitting models M2 and M7 with their corresponding number of fitting coefficients and their $\mathrm{R}^{2}$-value. Each coefficient $a_{0}, a_{1}, c, b$ and $k_{0}$ is fitted as a polynomial in the coupling parameter $\beta$ and the redshift $z$ (see Appendix A for further details). The $\mathrm{R}^{2}$-value is a measure of the goodness of fit: a value of 1 corresponds to a perfect fit, while 0 means that the model does not fit the data.

number of free parameters. Therefore, third order polynomials were the best compromise between complexity and goodness of fit.

The best fitting models were chosen according to their coefficient of determination (also known as $\mathrm{R}^{2}$-value), which is a statistical measure for the goodness-of-fit [66]. It can be simply defined as $R^{2}=1-S_{\text {res }} / S_{\text {tot }}$, where $S_{\text {res }}$ is the residual sum of squares (the residual between the data points and the fitting function) and $S_{t o t}$, which is the total sum of squares and is proportional to the variance of the data. An $\mathrm{R}^{2}$-value of 1 corresponds to a perfect fit. The analytical expressions for the best models M2 and M7 are shown in table III, together with their $\mathrm{R}^{2}$-value. We performed the whole Fisher analysis (see section VII below), for both of the models and the results on the parameter estimation are basically the same (less than half of a percent relative difference in the estimated final errors).

\section{Fitting functions and cosmological parameters}

In order to use the fitting formulae obtained before to forecast cosmological parameters of the model, we need to assume that the shape of the non-linear coupled DE signal does not change dramatically if the other cosmological parameters, apart from $\beta$ and $\sigma_{8}$, are modified by small amounts. This is justified since in the deeply non-linear regime, the evolution of perturbations is ruled by mode-mode coupling between high $k$ wavevectors (non-linear $k$ modes are not independent of each other anymore), which erases most of the information about initial conditions and makes the shape of the non-linear power spectrum at large $k$ practically independent of cosmological background parameters, such as $n_{s}, \Omega_{b}$ and $h$. This has been shown to be the case when calculating perturbatively non-linear corrections to the power spectrum, see for example [67, 68], but also analyzing the covariance matrix of non-linear power spectra using a large suite of N-body simulations as was investigated in [69]. Furthermore, the decoupling of virialized structures in the small scale regime from the background dynamics of the universe, is one of the cornerstones of the recently developed effective field theory of large-scale structure [70] and was also shown to be approximately true using a coarse grained cosmological perturbation approach [71]. Since $\beta$ and $\sigma_{8}$ are quantities that affect directly the linear perturbations and the virialization dynamics and we are only looking at a particular signal at very small scales, they should be the main parameters determining the shape of the non-linear "bump". An investigation of how robust these fitting formulas are, with respect to a change of cosmological parameters, would need either more high-resolution N-body simulation for coupled $\mathrm{DE}$ scenarios or the development of a consistent perturbation theory for modified gravity and scalar-tensor theories that reaches highly non-linear scales.

\section{NON-LINEAR POWER SPECTRUM AND ERROR ESTIMATION}

The accuracy of the fitting functions when compared to the original N-body simulation power spectra is shown in figure 2 . where the absolute value of the percentage error between fitting function and the original power spectra is plotted as a function of redshift and scale. In this case we show that for the model M7 the error remains well below $1 \%$ for a coupling constant of $\beta=0.05$ and for the scales and redshifts we are interested in. For higher couplings the error goes up to a maximum of $1.5 \%$. For the model M2 the same test gives similar results, yielding $<1 \%$ error in all interesting scales and redshifts. We can then expect that when applying these fitting functions on top of $\Lambda \mathrm{CDM}$ power spectra, the intrinsic error is less than $1 \%$ and the extra sources of error are all given by the N-body simulation spectra and by the estimators of the non-linear $\Lambda \mathrm{CDM}$ power spectrum.

Since the fitting functions shown in the previous section are useful when applied on top of a $\Lambda$ CDM non-linear power spectrum, we need to choose an estimator for the non-linear $\Lambda \mathrm{CDM} P(k)$. Our tests show that FrankenEmu [6], an improved version of the original Coyote Cosmic Emulator, performs better than the revised version of Halofit by [3], which is included in recent versions of CAMB [72]. While at $z=0$ both estimators work similarly well with an accuracy at the 5\% level in the BAO range, Halofit performs much worse with increasing redshift and increasing $k$, as illustrated in figure 3 . At $z=0$ the Cosmic Emulator shows a flat error curve for all scales up to the Nyquist frequency, below the error estimated for Halofit. A comparison between power spectrum estimators and N-body simulations has been performed also in [73], where they found similar results: Halofit and the Cosmic Emulator perform similarly in the BAO range, but the errors introduced by Halofit is above the $10 \%$ level at scales of 

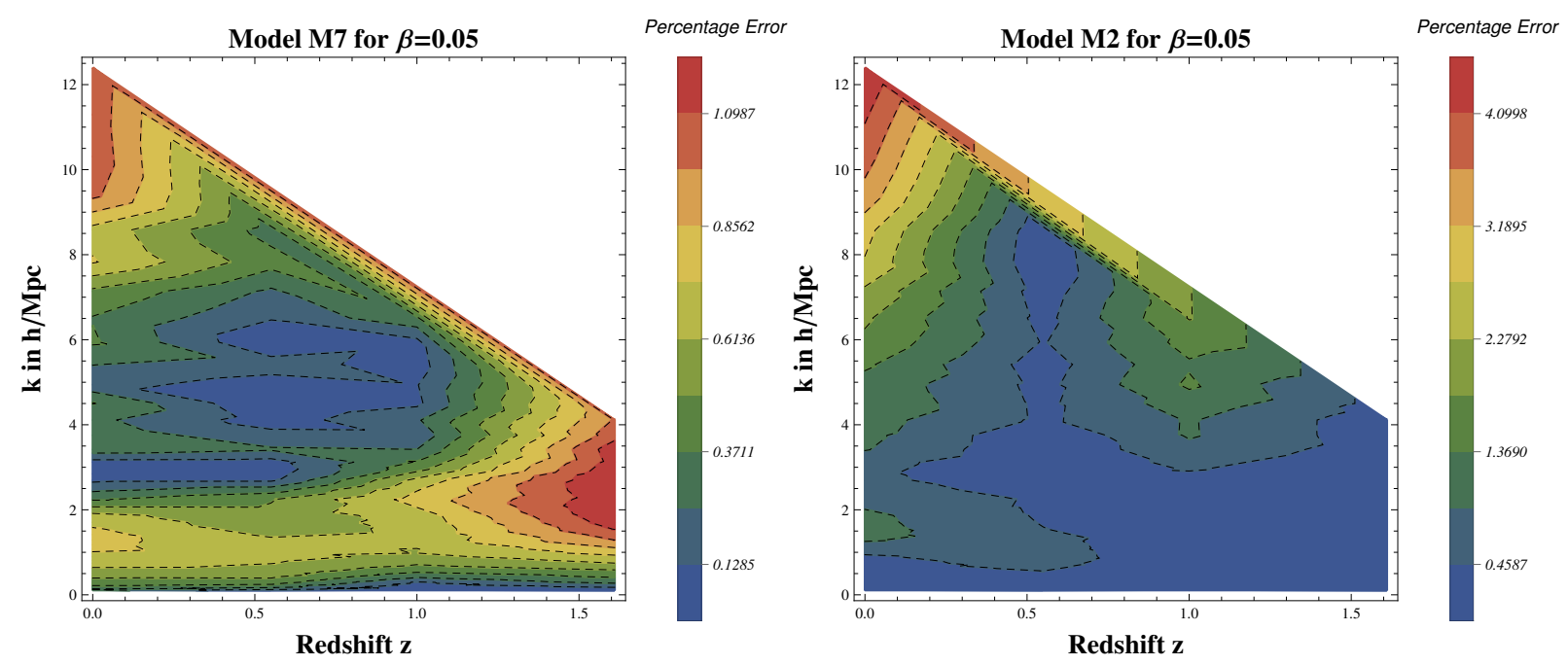

Figure 2. Error contour plot for the fitting functions of model M7 (left) and M2 (right) applied compared directly to the N-body simulations. We show that for the smallest coupling constant available in the simulations, $\beta=0.05$, the error remains below $1 \%$ for the scales and redshifts we are interested in.

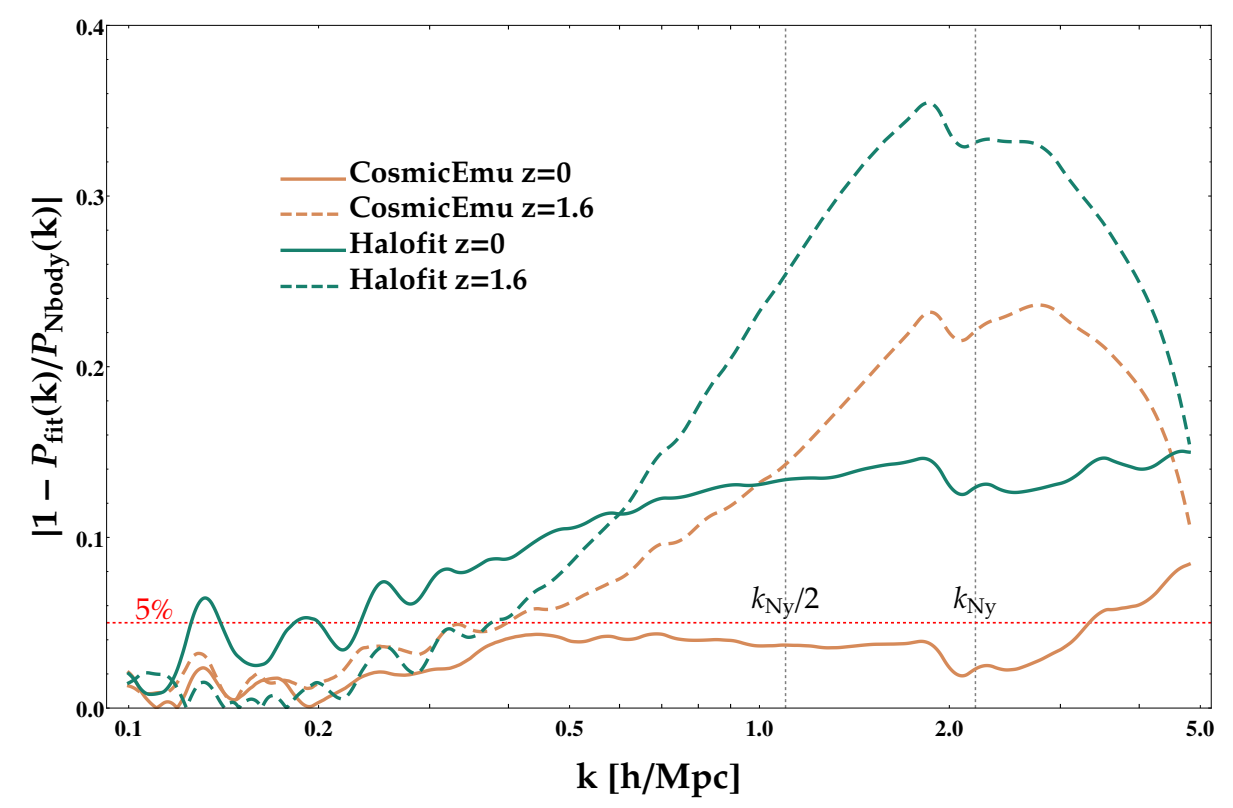

Figure 3. Relative error of the fitting functions Halofit (green) and CosmicEmulator (orange) (FrankenEmu) with respect to the CoDECS $\Lambda \mathrm{CDM} N$-body power spectra at two different redshifts (solid lines: $z=0$, dashed lines: $z=1.6$ ). While the relative error with respect to the CosmicEmulator remains below the $5 \%$ limit (horizontal red dashed line) for all interesting $k$-values, the error compared to Halofit is already bigger than $10 \%$ for $k \gtrsim 0.5 \mathrm{~h} / \mathrm{Mpc}$. Both the error of our simulation with respect to Halofit and to the CosmicEmulator increase as a function of redshift. The change of trend after the Nyquist frequency (marked with the vertical grey line) can be attributed to the use of the folding method for the CoDECS nonlinear power spectra.

around $k \approx 1 \mathrm{Mpc} / h$ and $z \gtrsim 1$. Therefore, we use the FrankenEmu as the preferred $\Lambda \mathrm{CDM}$ non-linear power spectrum estimator for our forecasting purposes. They claim to be accurate at the 1-3\% percent level around the scales of interest and they have performed very careful resolution tests using hundreds of realizations.

Another source of error is the sample variance error of the power spectrum when extracted from the N-body simulation. This depends on the number on the number $n_{\text {mod }}$ of independent modes available at each wavevector bin in $k$ and its given by [74]: $\sigma_{P}=\sqrt{\frac{2}{n_{\text {mod }}}} P(k)$. In figure 4 it can be seen as a blue shaded region marked by a red and blue solid line. For large scales 


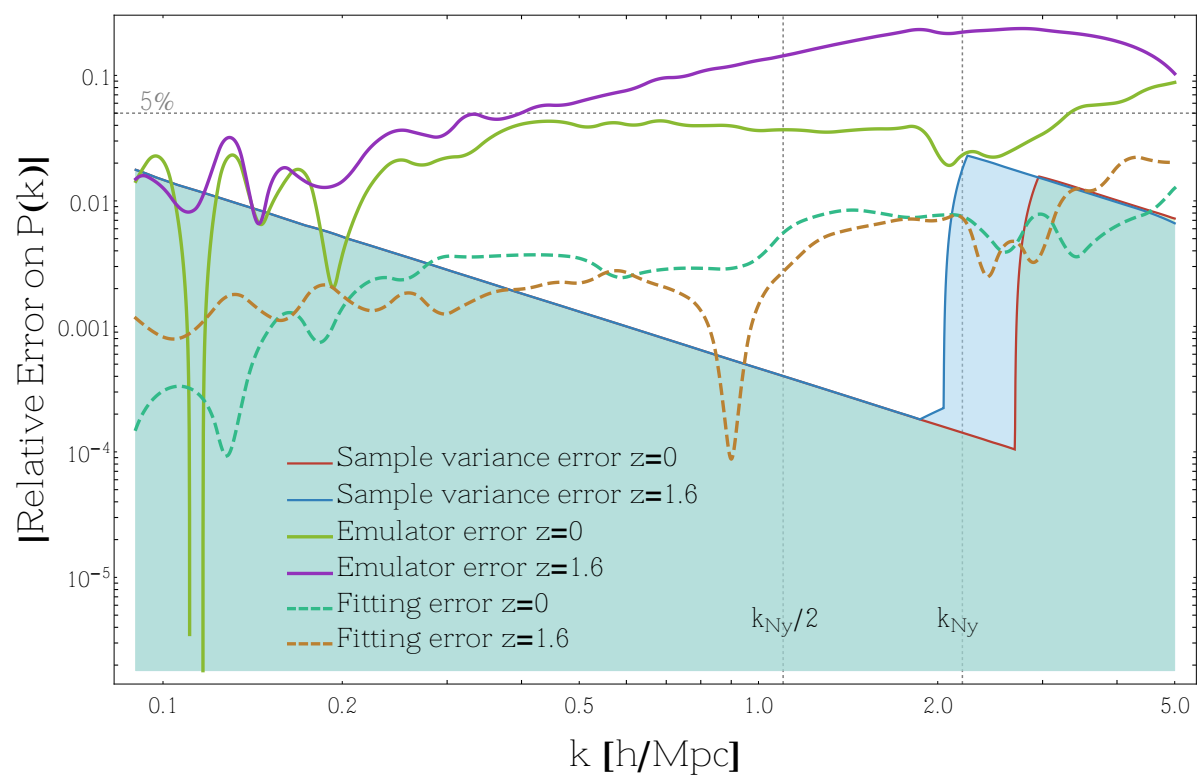

Figure 4. Different sources of error affecting the nonlinear power spectrum. Each source of error is shown at two different redshifts, $z=0$ and $z=1.6$. The sample variance error (red solid line and blue solid line, together with their shaded regions) corresponds to the error induced by the limited number of available $k$-modes when extracting the power spectrum; it has a sharp increase at the Nyquist frequency, since there the folded mesh has fewer modes available than the top level mesh, see section IV for more details. The fitting error (green and orange dashed lines) corresponds to the intrinsic error of the fitting functions with respect to an N-body simulation using the same parameters, calculated in section $\mathrm{V}$ The emulator error (green and purple thick lines) is the error of the Cosmic Emulator compared to a $\Lambda \mathrm{CDM} N$-body simulation from CoDECS (these two lines correspond to the orange lines of figure 33. The relative error increases with redshift and scale and reaches more than $15 \%$ at the Nyquist frequency for the highest redshift. The vertical grey-dashed lines mark the scales $k_{N y} / 2$ and $k_{N y}$.

this error is considerable, but there one usually uses just linear power spectra computed by Boltzmann codes, like CAMB or CLASS. At $k=0.1 h / \mathrm{Mpc}$ the binning error is at the percent level and decreases rapidly to negligible values, but then it increases again quickly at $k \sim 2 h / \mathrm{Mpc}$ since there the folded mesh for the power spectrum has again only few modes to sample from, as explained in section IV.

The intrinsic error of the fitting function is shown in figure 4 as a dashed green line and it remains well below the $1 \%$ level at all the scales of interest.

We include all errors discussed here in our Fisher forecast analysis. It turns out that they do not affect considerably the results for a survey like Euclid, which will measure such a high number of galaxies, that the sampling of the clustering signal will be not affected by small sources of noise in the data.

\section{FISHER MATRIX METHOD}

The Fisher matrix formalism ([75-77]) is one of the most popular tools to forecast the outcome of an experiment, because of its speed and its versatility when the likelihood is approximately Gaussian (see [78] for a method on how to improve this approximation). Here we apply the Fisher matrix formalism to two different probes, galaxy Clustering (GC) and weak lensing (WL), which are the ones that a future satellite like Euclid [48] will carry on board.

\section{A. Galaxy Clustering}

The Fisher matrix for the galaxy power spectrum has the following form [44, 76]:

$$
F_{i j}=\frac{V_{\text {survey }}}{8 \pi^{2}} \int_{-1}^{+1} \mathrm{~d} \mu \int_{k_{\min }}^{k_{\max }} \mathrm{d} k k^{2} \frac{\partial \ln P_{\text {obs }}(k, \mu, z)}{\partial \theta_{i}} \frac{\partial \ln P_{o b s}(k, \mu, z)}{\partial \theta_{j}}\left[\frac{n(z) P_{\text {obs }}(k, \mu, z)}{n(z) P_{o b s}(k, \mu, z)+1}\right]^{2} .
$$


Here $V_{\text {survey }}$ is the volume covered by the survey and contained in a redshift slice $\Delta z, n(z)$ is the galaxy number density as a function of redshift, $P_{o b s}(k, \mu, z)$ is the observed power spectrum as a function of the redshift $z$, the wavenumber $k$ and of $\mu \equiv \cos \alpha$, where $\alpha$ is the angle between the line of sight and the 3D-wavevector $\vec{k}$.

The derivatives in eq. 9 are taken with respect to a vector of cosmological parameters, $\theta_{i}=\left\{\beta^{2}, h, \log \mathscr{A}_{s}, n_{s}, \omega_{c}, \omega_{b}, b(z)\right\}$, where $b(z)$ is the galaxy bias. When we perform the numerical derivatives of $P_{o b s}(k, \mu, z)$, we have to take into account that not only the power spectrum and the background functions change, when the cosmological parameters are modified, but also $\mu$ and $k$ are changed by a geometrical factor depending on $H(z)$ and $D_{A}(z)$, due to the Alcock-Paczinsky effect [79].

The observed power spectrum contains all the cosmological information about the background and the matter perturbations as well as corrections due to redshift-space distortions and observational uncertainties. In this work we write it in the following form:

$$
P_{o b s}(k, \mu, z)=\frac{D_{A, f}^{2}(z) H(z)}{D_{A}^{2}(z) H_{f}(z)} b^{2}(z)\left(1+\beta_{d}(z) \mu^{2}\right)^{2} e^{-k^{2} \mu^{2}\left(\sigma_{r}^{2}+\sigma_{v}^{2}\right)} P(k, z)
$$

In the above formula, the subscript $f$ denotes the fiducial value of each quantity, $P(k, z)$ is the matter power spectrum, $D_{A}(z)$ is the angular diameter distance, $H(z)$ the Hubble function and $\beta_{d}(z)$ is the redshift space distortion factor, which in linear theory is given by $\beta_{d}(z)=f(z) / b(z)$, with $f(z) \equiv d \ln G / d \ln a$ representing the linear growth rate of matter perturbations. The exponential factor represents the damping of the observed power spectrum, due to two different effects: $\sigma_{z}$ an error induced by spectroscopic redshift measurement errors, which translates into a damping scale $\sigma_{r}=\sigma_{z} / H(z)$ and $\sigma_{v}$ which is the dispersion of pairwise peculiar velocities which are present at non-linear scales and also introduce a damping scale in the mapping between real and redshift space.

\section{Including sources of error in the Fisher formalism}

In section VI we discussed several sources of error affecting the non-linear power spectrum, the intrinsic errors of the coupled DE fitting functions, mode-binning errors in the N-body power spectrum and the estimation and interpolation error of the $\Lambda \mathrm{CDM}$ non-linear power spectrum obtained from the CosmicEmulator. We will take these sources of error into account in our Fisher forecast analysis by including them as extra noise affecting the observed power spectrum. The term in square brackets in eq. (9) corresponds to the inverse of the covariance $C=P(k, z)+n(z)^{-1}$. The "noise term" $n(z)^{-1}$ is the number density of sampling points for the matter power spectrum (galaxies in a survey), which gives us an estimate of the signal-to-noise ratio we can expect from the forecast: for a higher number density, the power spectrum is better sampled and more information can be extracted from it. In order to take into account the theoretical and numerical errors on $P(k, z)$, we decrease $n(z)$ by a factor that contains the relative errors on $P(k, z)$. In eq. 99, instead of $n(z)$, we then have an "effective" number density:

$$
n_{e f f}(k, z)=n(z) /\left(1+n(z) \tilde{\sigma}_{p}(k, z)\right)
$$

The term $\tilde{\sigma}_{p}(k, z)$ is a scale- and redshift-dependent term which is the square root of the sum of the relative errors squared. We take into account all error sources which affect $P(k, z)$ due to different reasons, as explained in section VI One of them is the difference between our power spectrum estimator and the $\mathrm{N}$-body simulation: $\sigma_{p}(k, z)=\left(P_{\text {numerical }}(k, z)-P_{\text {true }}(k, z)\right) / P_{\text {true }}(k, z)$. If $\tilde{\sigma}_{p}(k, z)=0$ or it is negligible, the effective number density will be the observed one $n_{\text {eff }}(z)=n(z)$; otherwise, $n_{e f f}(z)<n(z)$, the effective number of sampling points being reduced, together with the amount of information one can extract from the power spectrum. As long as $n(z) P(k, z) \gg 1$ for all $z$ and $k$, the sampling will be always good enough to extract cosmological information from the power spectrum even in the presence of noise. For the specifications used in this work (see table $\mathrm{V}$ below), $n(z) P(k, z)$ is larger than 1 in all scales of interest and therefore the theoretical and numerical error on $P(k, z)$ does not have such a considerable effect on the parameter estimation, as one would expect naively. We test the inclusion of the effective number density $n_{e f f}(z)$ on the Fisher forecast analysis and we find that the relative 1- $\sigma$ marginalised errors on each cosmological parameter are between $8 \%$ and $15 \%$ higher when using the estimated uncertainties on the power spectrum.

\section{Systematic bias on cosmological parameters}

In this section we will quantify the effect of the systematic errors due to the uncertainties on the non-linear power spectrum. We will show how big this systematic bias would be, if we used for our forecasts a power spectrum which is not the "correct" one, for example one obtained from a single N-body realization. For the following discussion on systematic biases, we will use the expressions derived in the Appendix B of [80]. 
The linear bias on a cosmological parameter $\delta \theta_{i}$ due to the bias $\delta \psi_{i}$ in a parameter of the model which we assume fixed and cannot be measured is given by:

$$
\delta \theta_{i}=-\left[F^{\theta \theta}\right]_{i k}^{-1} F_{k j}^{\theta \psi} \delta \psi_{j}
$$

In our case we will have only one systematic parameter $\psi$, which controls the difference between the "true" power spectrum $P_{\text {true }}$ and and our simulated power spectrum $P_{\text {num }}$ :

$$
P_{\psi}=\psi P_{\text {num }}+(1-\psi) P_{\text {true }}
$$

$\psi$ can vary continously so that for $\psi=1$ we recover $P_{\text {num }}$, while for $\psi=0$ we obtain $P_{\text {true }}$. We can define the relative difference between $P_{\text {true }}$ and $P_{\text {num }}$ as:

$$
\sigma_{p}(k, z) \equiv \frac{P_{\text {num }}(k, z)-P_{\text {true }}(k, z)}{P_{\text {true }}(k, z)}
$$

The $F^{\theta \theta}$ in eqn 12 above is simply the usual Fisher matrix:

$$
F^{\theta \theta}=\frac{1}{2} \operatorname{tr}\left(C^{-1} \partial_{i}^{\theta} C C^{-1} \partial_{j}^{\theta} C\right)
$$

while the pseudo-Fisher matrix between measured and assumed parameters $F^{\theta \psi}$ is:

$$
F_{i j}^{\theta \psi}=\frac{1}{2} \operatorname{tr}\left(C^{-1} \partial_{i}^{\theta} C C^{-1} \partial_{j}^{\psi} C\right)
$$

which for one systematic parameter only, is just a column vector.

In the case of galaxy Clustering we will compute $F^{\theta \psi}$ in the following way, using the fact that for $\psi=1, C=P_{n u m}(k, z)+$ $n^{-1}(z)$ and $\left.P_{\psi}\right|_{\psi=1}=P_{n u m}$ :

$$
\left.F_{i}^{\theta \psi} \propto \int \mathrm{d} k k^{2}\left(\frac{n_{\text {eff }}(z) P_{\text {num }}(k, z)}{n_{\text {eff }}(z) P_{\text {num }}(k, z)+1}\right)^{2}\left(\frac{1}{P_{\text {num }}(k, z)}\right)^{2} \frac{\partial P_{\psi}}{\partial \psi}\right|_{\psi=1} \frac{\partial P_{\text {num }}}{\partial \theta_{i}}
$$

in this step we have assumed that we have no systematic parameters affecting the galaxy number density $n(z)$ and therefore, its derivative w.r.t $\psi$ vanishes. Also, for notational simplicity we left out the integral over $\mu$ and the form of the observed power spectrum.

We have then $\partial_{\psi} P_{\psi}=-P_{\text {true }}+P_{\text {num }}=P_{\text {true }} \sigma_{p}(k, z)$ and we just need to perform eqn. 15 with eqn. 12 in order to obtain the systematic biases on the cosmological parameters.

In the following table $[\mathrm{IV}$ we present the results on the systematic bias, for a standard $\Lambda \mathrm{CDM}$ forecast, for different maximum $k$ coverages, up to a maximum $k$ of $1.1 \mathrm{~h} / \mathrm{Mpc}$. We will regard as a "true" power spectrum $P_{\text {true }}$, the one obtained by the Cosmic (Franken) Emulator [6], since they have performed a careful analysis of resolution effects using a large set of simulations and claim to be accurate for $k<1.0 \mathrm{~h} / \mathrm{Mpc}$ at the $1 \%$ percent level compared to state of the art N-body simulations. On the other hand, the numerical "biased" power spectrum $P_{n u m}$, is the one obtained from the CoDECS $\Lambda$ CDM run, which consists on only one realization. We have left out the $\mathrm{CDE}$ coupling parameter $\beta$, since in that case we do not have any other prediction in the non-linear regime to compare with.

\begin{tabular}{|c|c|c|c|c|c|}
\hline Parameter & $h$ & $\ln \mathscr{A}_{s}$ & $n_{s}$ & $\omega_{b}$ & $\omega_{c}$ \\
\hline fiducial & 0.7036 & 2.42 & 0.966 & 0.04503 & 0.2256 \\
\hline $\begin{array}{c}\mathbf{k}_{\mathbf{m a x}}=\mathbf{k}_{\mathbf{N y}} / \mathbf{2}=\mathbf{1 . 1 h} / \mathbf{M p c} \\
\text { syst. bias with } \delta \psi=1\end{array}$ & -0.0016 & -0.15 & 0.061 & 0.0028 & -0.0031 \\
\hline $\mathbf{k}_{\mathbf{m a x}}=\mathbf{0 . 3 5} \mathbf{h} \mathbf{M p c}$ & & & & & \\
\hline syst. bias with $\delta \psi=1$ & -0.0094 & -0.11 & 0.045 & 0.0021 & -0.0039 \\
\hline $\mathbf{k}_{\mathbf{m a x}}=\mathbf{0 . 1 5 h} / \mathbf{M p c}$ & & & & & \\
\hline syst. bias with $\delta \psi=1$ & -0.0032 & -0.04 & 0.018 & 0.00026 & -0.0024 \\
\hline
\end{tabular}

Table IV. Systematic bias on the $\Lambda$ CDM cosmological parameters evaluated at our fiducial model. The systematic bias is higher when probing smaller scales, where uncertainties in the non-linear power spectrum are higher. This highlights the importance of modelling correctly the non-linear power spectrum in order to analyze data. Using the wrong non-linear power spectrum produces statistical errors which are larger or of the same order as the statistical results. 
The results of table IV show how important it is to model accurately the non-linear power spectrum in order to make forecasts and to analyze the upcoming data of large scale structure surveys like Euclid [48]. The systematic errors on the cosmological parameters can be bigger than the statistical errors (compare to table VI and figure 5 in the results section below). This is the case if, as in our example scenario, we would use a non-linear power spectrum that is inaccurate by about $10-15 \%$ in the nonlinear regime at higher redshifts (which was shown in figure 3). Therefore, it is well justified to choose for our Fisher forecasts the Cosmic Emulator as the "true" $\Lambda \mathrm{CDM}$ non-linear power spectrum estimator, since this is the most accurate predictor up to date. It would still be interesting to know how robust is the signal of the coupling parameter $\beta$ with respect to changes in the other parameters or in the $\Lambda$ CDM non-linear prediction, but as long as we do not have better and faster semianalytic methods applicable to general models of dark energy, the estimation of systematic biases of extra parameters is an impossible task.

\section{Choice of the $k_{\text {max }}$ integration limit in the Fisher formalism}

There are at least three ways of setting the maximum $k$ mode used in the Fisher forecast integration (eqn. 9). One common choice is to set a hard cut in $k$ at all redshifts, and depending if one wants to include or not non-linear effects, this cut can be taken at linear scales, smaller than $k=0.1 \mathrm{~h} / \mathrm{Mpc}$, or at non-linear scales $k>0.1 \mathrm{~h} / \mathrm{Mpc}$. In the latter case if one is using a power spectrum calculated in linear theory, one needs to to use some Lagrangian damping correction, as introduced originally in [76, 81], in order to take into account broadening effects on the BAO peak induced by the non-linear evolution of densities and velocities. Another option to cut off the power spectrum is to demand that the variance $\sigma_{8}(z ; k)$ stays below a specified value at each redshift, therefore implicitly changing the cutoff in $k$ as a function of $z$. An usual choice for this is $\sigma_{8}\left(z ; k_{c u t}\right)=0.25$, as was done previously in [82]. Since we are assuming to have a knowledge of the non-linear power spectrum up to the Nyquist frequency, we implement for our forecasts a hard-cut method, at $k_{N y}$ and at $k_{N y} / 2$, without the need of any Lagragian damping correction. However, to be conservative, we cite as our main results the ones in which the cut is performed at $k_{N y} / 2$ in order to eliminate any possible unknown contribution from the numerical high-frequency noise entering the estimation of $P(k)$ at the Nyquist frequency (see e.g. [63, 83] for similar prescriptions on where to cut the non-linear power spectrum).

\section{B. Weak Lensing}

For the weak lensing power spectrum the Fisher matrix is just a sum over all possible correlations at different redshift bins [75], namely:

$$
F_{\alpha \beta}=f_{s k y} \sum_{\ell}^{\ell_{\max }} \sum_{i, j, k, m} \frac{(2 \ell+1) \Delta \ell}{2} \frac{\partial P_{i j}(\ell)}{\partial \theta_{\alpha}} C_{j k}^{-1} \frac{\partial P_{k m}(\ell)}{\partial \theta_{\beta}} C_{m i}^{-1}
$$

With the power spectrum of the shear field given by:

$$
P_{i j}(\ell)=\frac{9}{4} \int_{0}^{\infty} \mathrm{d} z \frac{W_{i}(z) W_{j}(z) H^{3}(z) \Omega_{m}^{2}(z)}{(1+z)^{4}} P_{m}(\ell / r(z))
$$

where $P_{m}$ is the matter power spectrum discussed above, the indices $i, j$ stand for each of the $\mathscr{N}$ redshift bins and the window functions are given by:

$$
W(z)=\int_{z}^{\infty} \mathrm{d} \tilde{z}\left(1-\frac{r(z)}{r(\tilde{z})}\right) n(\tilde{z})
$$

where the normalized galaxy distribution function is:

$$
n(z)=z^{2} \exp \left(-\left(z / z_{0}\right)^{3 / 2}\right)
$$

Here the median redshift and $z_{0}$ are related by $z_{m e d} \approx 1.412 z_{0}$. The corresponding covariance matrix has the following form:

$$
C_{i j}(\ell)=P_{i j}(\ell)+\delta_{i j} \gamma_{i n t}^{2} n_{i}^{-1}+K_{i j}(\ell)
$$

where $\gamma_{\text {int }}$ is the intrinsic galaxy ellipticity and the shot noise term contains 


$$
n_{i}=3600\left(\frac{180}{\pi}\right)^{2} n_{\theta} / \mathscr{N}
$$

with $n_{\theta}$ the total number of galaxies per $\operatorname{arcmin}^{2}$ assuming that the redshift bins have been chosen such that each contain the same amount of galaxies (equi-populated bins). The matrix $K_{i j}(\ell)$ is a diagonal "cutoff" matrix whose entries increase to very high values at the scales where the power spectrum $P(k)$ has to be cut to avoid the inclusion of numerical noise errors. Since $K_{i j}(\ell)$ depends on the multipole $\ell$, which itself depends on $k$ and $z$ through: $\ell=k r(z)$, each of the entries $K_{i i}$ increase with the multipole in a different way. For our purposes we chose a very sharp cutoff, such that multipoles containing wavenumbers $k$ beyond the Nyquist frequency (or half of the Nyquist frequency, for our reference case) at the center of the redshift bin $i$, do not contribute to the Fisher matrix at all after the corresponding $\ell_{\text {cut }}$ (specified in table VIII below).

\section{Adding non-linear corrections to the power spectrum}

We are interested in a Fisher forecast that includes information from non-linearities. In this case we cannot separate the power spectrum, into a power spectrum at redshift zero multiplied by the square of the normalized growth factor, but we need to evaluate directly the non-linear power spectra $P(k, z)$ at different redshifts. A full non-linear correction for the redshift space distortions would be also desirable, but since the modelling of that effect is yet to be understood in general cases, we use as a first approximation an exponential damping of the form $\exp \left(-k^{2} \mu^{2} \sigma_{v}^{2}\right)$, where $\sigma_{v}$ is the pairwise peculiar velocity of galaxies induced by non-linearities in the matter and velocity power spectrum. This is the first term of a set of corrections that can be applied to the Kaiser formula [84] for the clustering in redshift space (see e.g. [85], [86], [87], [88]). We use a value of $\sigma_{v}=300 \mathrm{~km} / \mathrm{s}$ which is an approximate and conservative value based on the estimations by [85], in which the authors test a variety of redshift-space distortion models.

As already mentioned, the damping term, introduced by [76], which should correct the linear $P(k)$, especially the position of the BAO peaks, for non-linearities, is not included here, since we assume that we have a complete model of the non-linear power spectrum and therefore all possible corrections are already included in our fitting functions and power spectrum emulators.

In order to implement our model of coupled Dark Energy, we use for $H(z)$ and $D(z)$ tables precomputed using a modified version of CAMB, that includes the exponential and inverse-power law potentials for coupled dark energy [37, 82]. We calculate these tables for each of the parameters $\theta_{i} \pm \varepsilon$, where $\varepsilon=0.03$. We do the same for the linear perturbation quantity $G(z)$, whose logarithmic derivative with respect to $\ln (a)$, known as the growth rate $f(z)$ enters the redshift space distortion term in 10 . The background quantities $H(z)$ and $D(z)$ are important in the Fisher forecast for the Alcock-Paczynski geometrical term, which we take into account in the observed power spectrum.

The full non-linear power spectrum we use in our method is then obtained as follows.

- At linear scales $k \lesssim 0.1 \mathrm{~h} / \mathrm{Mpc}$ the linear power spectrum is obtained from our modified version of CAMB which includes the effect of the DM-DE coupling.

- At non-linear scales, $k \gtrsim 0.1 \mathrm{~h} / \mathrm{Mpc}$, we use a combination of the power spectrum calculated with the cosmic emulator FrankenEmu and our fitting formulas for CDE that account for the non-linear dynamics in presence of a fifth force.

- The matching at $k \approx 0.1 \mathrm{~h} / \mathrm{Mpc}$ is performed using different interpolation methods, either averaging on both sides and smoothing out or allowing for a small discontinuity. The matching point is left out of the Fisher integral and we check for this effect, showing that the different methods have a negligible effect (less than 2 percent) on the final absolute errors on the parameters.

- In order to be conservative in terms of numerical errors and noise, we cut the power spectra at half of the Nyquist frequency (we also test and compare with a cutoff at $k_{N y}$ ) and we include all sources of errors specified in section VII A 1, as effective noise terms into our Fisher estimation.

\section{RESULTS}

We now present results for galaxy clustering and weak lensing, using the specifications for a Euclid-like survey as described in tables $\mathrm{V}$ and VII below. We use the Fisher formalism described in section VII to forecast the errors in the cosmological parameters, using information from the non-linear power spectrum for coupled Dark Energy models, as obtained with the procedure described in section $[\mathrm{IV}]$ together with the fitting functions obtained in section $\mathrm{V}$ To make our estimation more realistic, we also take into account all sources of error and systematics for the power spectrum which were discussed in section VI As a way of testing our improvement on the parameter estimation, we also perform two extra Fisher forecasts, first using only linear 


\begin{tabular}{|c|c|c|}
\hline Parameter & Value & Description \\
\hline$A_{\text {survey }}$ & $15000 \mathrm{deg}^{2}$ & Survey area in the sky \\
$\sigma_{z}$ & 0.001 & Photometric redshift error \\
$\sigma_{v}$ & $300 \mathrm{~km} / \mathrm{s}$ & Fiducial pairwise velocity dispersion \\
$\Delta z$ & 0.2 & Redshift bin width \\
$\left\{z_{\min }, z_{\max }\right\}$ & $\{0.6,2.0\}$ & Min. and max. limits for redshift bins \\
\hline
\end{tabular}

Table V. Specifications for the Fisher Matrix of an Euclid-like galaxy clustering survey.

\begin{tabular}{|c|c|c|c|c|c|c|}
\hline Parameter & $\beta^{2}$ & $h$ & $10^{9} \mathscr{A}_{s}$ & $n_{s}$ & $\Omega_{b}$ & $\Omega_{c}$ \\
\hline fiducial & 0.0025 & 0.7036 & 2.42 & 0.966 & 0.04503 & 0.2256 \\
\hline $\mathbf{k}_{\mathbf{m a x}}=\mathbf{k}_{\mathbf{N y}} / \mathbf{2}$ & & & & & & \\
\hline abs. error & 0.000346 & 0.00160 & 0.01855 & 0.00267 & 0.00084 & 0.00088 \\
relative error & $14 \%$ & $0.23 \%$ & $0.77 \%$ & $0.28 \%$ & $1.9 \%$ & $0.39 \%$ \\
\hline $\mathbf{k}_{\mathbf{m a x}}=\mathbf{k}_{\mathbf{N y}}$ & & & & & & \\
\hline abs. error & 0.000305 & 0.00151 & 0.01808 & 0.00249 & 0.00081 & 0.00085 \\
relative error & $12 \%$ & $0.22 \%$ & $0.75 \%$ & $0.26 \%$ & $1.8 \%$ & $0.38 \%$ \\
\hline
\end{tabular}

Table VI. 1- $\sigma$ fully marginalized errors on the cosmological parameters of the model for a galaxy clustering Fisher forecast. Two cases are presented, a hard cutoff of the power spectrum at $k_{\max }=k_{N y} / 2$, and a hard cut at $k_{N y}$.

power spectra for the CDE model and then correcting these linear $P(k)$ with the latest version of Halofit [3], which was designed for $\Lambda \mathrm{CDM}$ only.

The fiducial parameters are $\omega_{c}=0.1117, \omega_{b}=0.0223, n_{s}=0.966, \log \mathscr{A}_{s}=-19.8395, h=0.7036, \beta^{2}=0.0025$, which are consistent with WMAP7 results (see table III). The fiducial values for the galaxy bias $b(z)$ used for the GC probe are taken from the Euclid specifications (see [47, 48, 89]) For our final results, we convert these parameters into the set $p_{i}=$ $\left\{\beta^{2}, h, 10^{9} \mathscr{A}_{s}, n_{s}, \Omega_{c}, \Omega_{b}\right\}$ marginalizing over the bias $b(z)$ (for the GC case) and using a Jacobian transformation to convert into the new set of parameters, which is allowed by the Fisher matrix formalism. We choose to forecast the error on the square of the coupling parameter $\beta^{2}$, because this is the quantity entering the modified gravitational Newton constant $G_{e f f}$ in the limit of linear perturbations (cf. eq. 7), therefore giving the strength of the "fifth force". The corresponding fiducial value for the coupling, $\beta=0.05$, is still compatible with recent limits set by analyzing the data from the Planck Satellite (see [37, 40]).

\section{A. Galaxy clustering}

Table $\mathrm{V}$ shows the specifications of a Euclid-like survey, which are used in our Fisher forecast. While Euclid specifications use 14 redshift bins of a width $\Delta z=0.1$ (see [48], table 3 in that work), we use only 6 bins of a width of 0.2 . We check in the case of $\Lambda \mathrm{CDM}$, that this re-binning ( done using the specified number of galaxies and the corresponding comoving volumes in our cosmology) has a very small effect (of a few percent) on the estimation of the 1- $\sigma$ errors on the parameters.

In table $\mathrm{VI}$ we show the fully marginalized $1-\sigma$ errors on the final cosmological parameters $p_{i}=\left\{\beta^{2}, h, 10^{9} \mathscr{A}_{s}, n_{s}, \Omega_{c}, \Omega_{b}\right\}$, performing the non-linear power spectrum cut-off at two different scales: $k_{\max }=k_{N y} / 2$ and $k_{\max }=k_{N y}$. As explained above, we take as a reference result the one corresponding to a cutoff at $k_{N y} / 2$. The gain in constraining the $\beta^{2}$ parameter when going from $k_{N y} / 2$ to $k_{N y}$ is of two percent points in the relative errors, while for the other cosmological parameters the improvement is negligible.

\section{Variation of the $k_{\max }$ integration limit}

We now test the gain in information obtained by including progressively more non-linear wavemodes $k$ into the Fisher integration. We perform the same Fisher forecast, each time increasing the maximum mode $k_{\max }$ at which the integration is cut off. In figure 5 we show how the $1-\sigma$ fully marginalized error on the cosmological parameters $p_{i}$ changes with an increase of $k_{\max }$. The error decreases steadily with an increase of $k_{\max }$, where the biggest gain is achieved when going from linear $(k \approx 0.1 \mathrm{~h} / \mathrm{Mpc})$ to mildly non-linear ( $k \approx 0.3 h / \mathrm{Mpc}$ ) scales. For parameters like $h$ and $\Omega_{b}$, an approximate plateau is reached already before $k_{\max } \approx 1.0$, while for $\beta^{2}$ there is still a considerable gain in parameter constraints when going into smaller scales, even beyond $k_{N y} / 2$ (consistent with table VI above). This happens, qualitatively, because at small scales we have a well-defined characteristic 


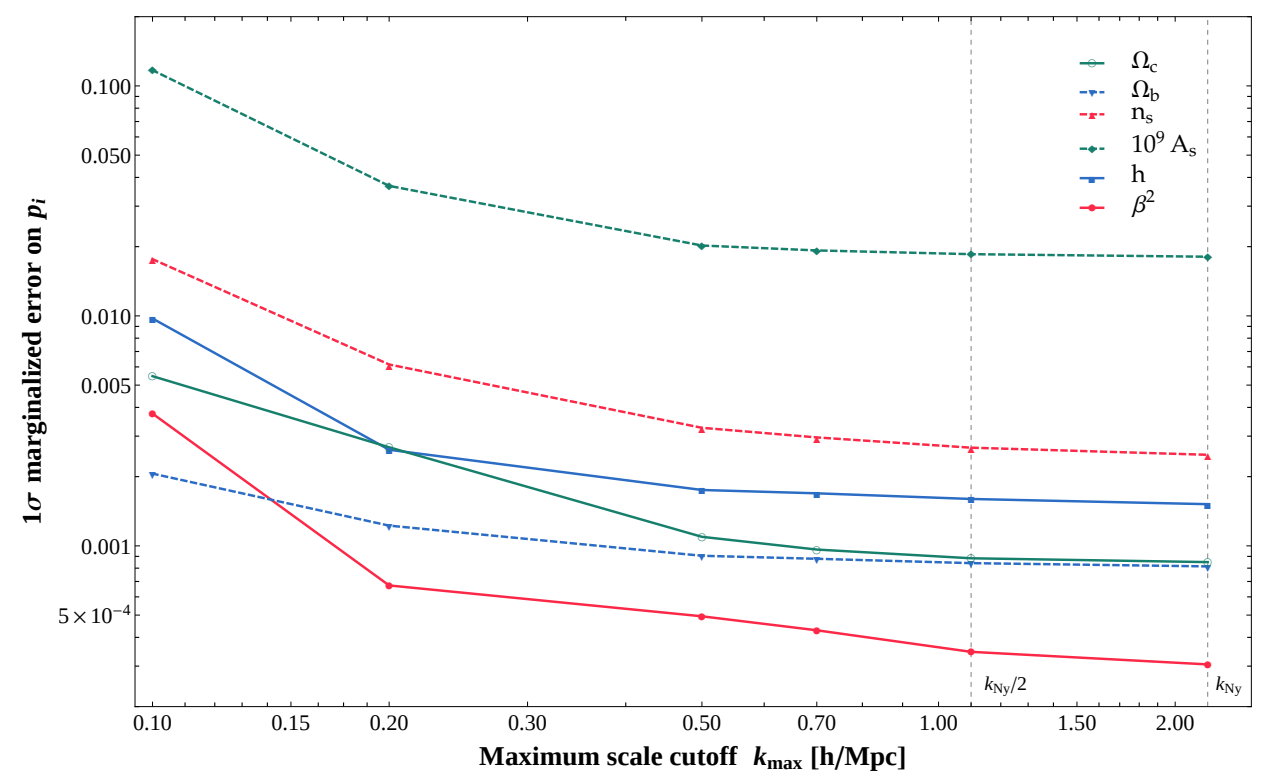

Figure 5. Change of the 1- $\sigma$ fully marginalized error on the set of cosmological parameters $p_{i}$ for a galaxy clustering Fisher forecast, as a function of the maximum mode $k_{\max }$ used as a cutoff in the Fisher matrix integration. When increasing the maximum $k_{\max }$ the errors on the parameters get steadily smaller, especially when going from linear $(k \approx 0.1 h / \mathrm{Mpc})$ to mildly non-linear $(k \approx 0.3 h / \mathrm{Mpc})$ scales. The vertical dashed grey lines, mark the half and the full Nyquist frequencies.
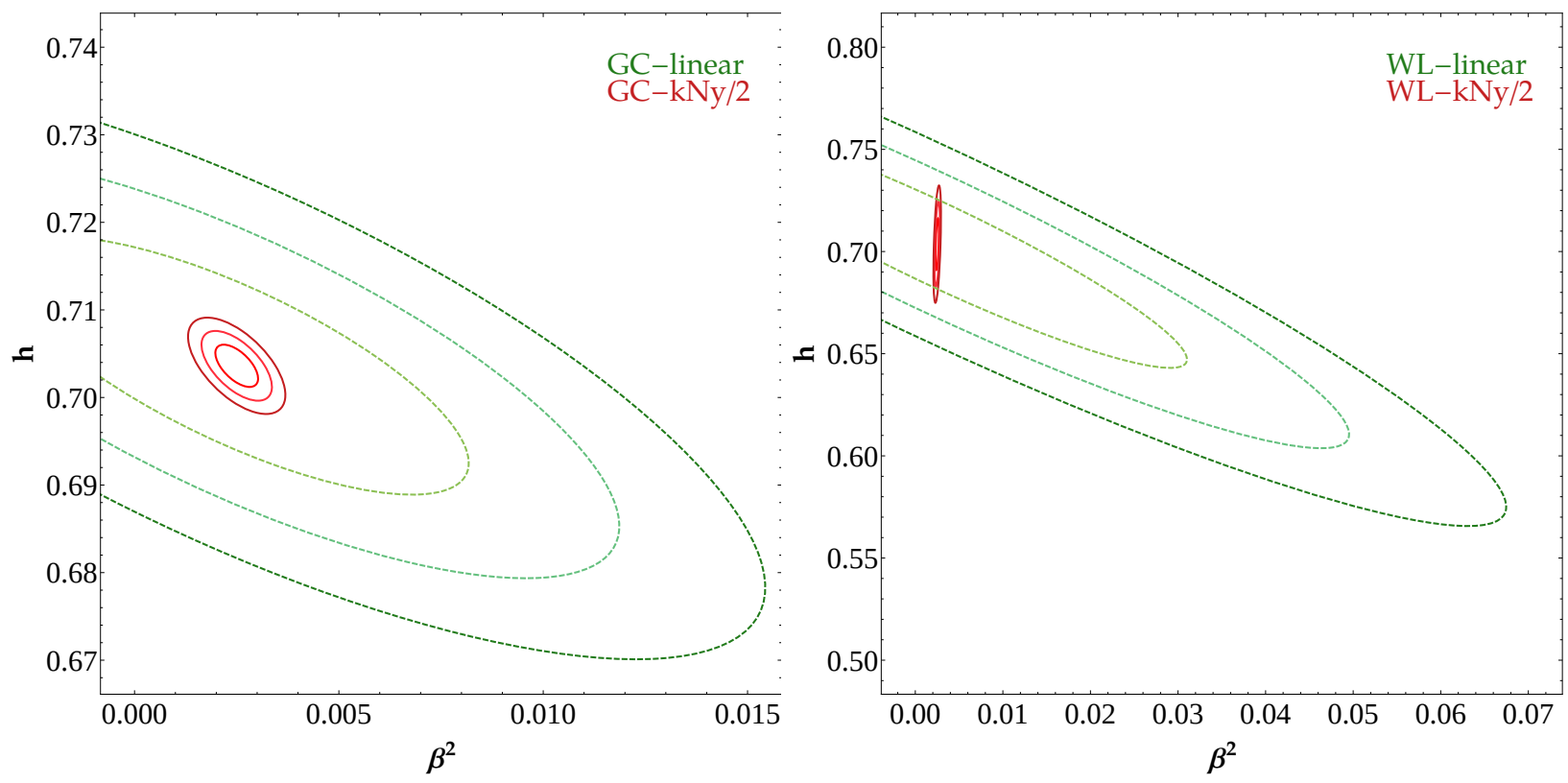

Figure 6. Marginalized confidence contour regions 1,2,3- $\sigma$ for $\beta^{2}$ and $h$. The plots correspond to a comparison between linear (green-dashed lines) and non-linear (red-solid lines) scales for GC (left) and WL (right) using the scale cutoff at $k_{N y} / 2$.

signal coming from non-linear interactions including the fifth force which itself involves the $\beta$ coupling, while the information on the backgorund cosmological parameters gets washed out (c.f. section $\mathrm{VC}$ above). 


\begin{tabular}{|c|c|c|}
\hline Parameter & Value & Description \\
\hline$f_{s k y}$ & 0.364 & Observed sky fraction \\
$\gamma_{i n t}$ & 0.22 & Intrinsic alignment \\
$n_{\theta}$ & 30 & Galaxy density per arcmin $^{2}$ \\
$\sigma_{p z}$ & 0.05 & Photometric redshift error \\
\hline
\end{tabular}

Table VII. Specifications for the Fisher Matrix of an Euclid-like weak lensing survey.

\begin{tabular}{|c|c|c|}
\hline Parameter & Value & Description \\
\hline $\mathscr{N}$ & 6 & Number of redshift bins \\
$z_{\text {peak }}$ & $\{0.59,0.75,0.90,1.06,1.28,1.57\}$ & $z$-position of peak of the bin \\
$w_{z}$ & $\{0.22,0.23,0.25,0.27,0.32,0.51\}$ & full width at half maximum of the peak \\
$\ell_{\text {cut }, k_{1}}$ & $\{1686,2070,2410,2753,3155,4344\}$ & cutoff in multipole $\ell$ at the center of each bin for $k_{\max }=k_{N y} / 2$ \\
$\ell_{\text {cut }, k_{2}}$ & $\{3372,4141,4820,5506,6311,8689\}$ & cutoff in multipole $\ell$ at the center of each bin for $k_{\max }=k_{N y}$ \\
$z_{\text {range }}$ & $0.5 \leq z \leq 3$ & Total range in redshift of each bin \\
\hline
\end{tabular}

Table VIII. Redshift bins specifications for an Euclid-like weak lensing survey using equipopulated redshift bins in the range $0 \leq z \leq 3$ and the corresponding values for the cutoff applied in the multipoles $\ell$ at each redshift bin, for two different cases: scales larger than $k_{1}=k_{N y} / 2$ and scales larger than $k_{2}=k_{N y}$.

\section{B. Weak lensing}

Table VII show the specifications for a weak lensing probe in an Euclid-like survey. The redshift bins are chosen in such a way that they contain an equal number of galaxies (equipopulated bins). The bins are then given by:

$$
n_{i}(z)=\frac{1}{2} n(z)\left[\operatorname{Erf}\left(\frac{\tilde{z}_{i+1}-z}{\sigma_{p z} \sqrt{2}}\right)-\operatorname{Erf}\left(\frac{\tilde{z}_{i}-z}{\sigma_{p z} \sqrt{2}}\right)\right]
$$

where $\tilde{z}_{i}$ are the values of the bin intervals in the range $z_{\text {range }}=0.5 \leq z \leq 3$ chosen such that for each interval the integral over the galaxy distribution function $n(z)$ (eqn. 19 is equal. The resulting peaks of the bins and their full width at half maximum are specified in table VIII.

Analogously to the galaxy clustering case, we show in table IX the fully marginalised 1- $\sigma$ errors on the parameters $p_{i}$. The sum over multipoles $\ell$ in eq. (16) is performed from $\ell_{\min }=5$ up to a maximum of $\ell_{\max }=20000$, but as explained in section VII B we perform a cutoff at each redshift bin, so that no scales in the non-linear power spectrum beyond the half of the Nyquist (for our reference case) or beyond the Nyquist frequency (for our second case) contribute to the WL signal. The values of these cutoffs, $\ell_{c u t}$ for the two different cases $k_{1}=k_{N y} / 2$ and $k_{2}=k_{N y}$ are listed in table VIII. In contrast to the GC case, going from $k_{N y} / 2$ to $k_{N y}$ in a WL survey does bring a noticeable improvement on the estimation of parameters.

The dependence of the $1-\sigma$ fully marginalized error on each parameter $p_{i}$ with respect to $\ell_{\max }$ is shown in figure 7 . When using just linear power spectra information, the error on the parameters does not improve if one increases the scale $\ell_{\text {max }}$, while

\begin{tabular}{|c|c|c|c|c|c|c|}
\hline Parameter & $\beta^{2}$ & $h$ & $10^{9} \mathscr{A}_{S}$ & $n_{s}$ & $\Omega_{b}$ & $\Omega_{c}$ \\
\hline fiducial & 0.0025 & 0.7036 & 2.42 & 0.966 & 0.04503 & 0.2256 \\
\hline$\ell_{\mathbf{c u t}, \mathbf{k}_{\mathbf{N y}} / \mathbf{2}}$ & & & & & & \\
\hline abs. error & 0.000125 & 0.00835 & 0.112 & 0.0105 & 0.0032 & 0.0046 \\
relative errror & $5.0 \%$ & $1.2 \%$ & $4.6 \%$ & $1.1 \%$ & $7.1 \%$ & $2.0 \%$ \\
\hline$\ell_{\mathbf{c u t}_{\mathbf{k}} \mathbf{k}_{\mathbf{N y}}}$ & & & & & & \\
\hline abs. error & 0.000097 & 0.0068 & 0.058 & 0.0085 & 0.0022 & 0.0032 \\
relative error & $3.9 \%$ & $0.97 \%$ & $2.4 \%$ & $0.88 \%$ & $4.9 \%$ & $1.4 \%$ \\
\hline
\end{tabular}

Table IX. 1- $\sigma$ fully marginalized errors on the cosmological parameters of the model for a weak lensing Fisher forecast. Two cases are presented, a redshift-dependent cutoff $\ell_{c u t, k_{N y} / 2}$ and a cutoff $\ell_{c u t, k_{N y}}$, corresponding to cutting off the non-linear power spectrum at the half and at the full Nyquist frequency (analogously to the GC case) as explained in the main text. As opposed to the GC case, going into smaller scales in a WL survey does bring a noticeable improvement. 


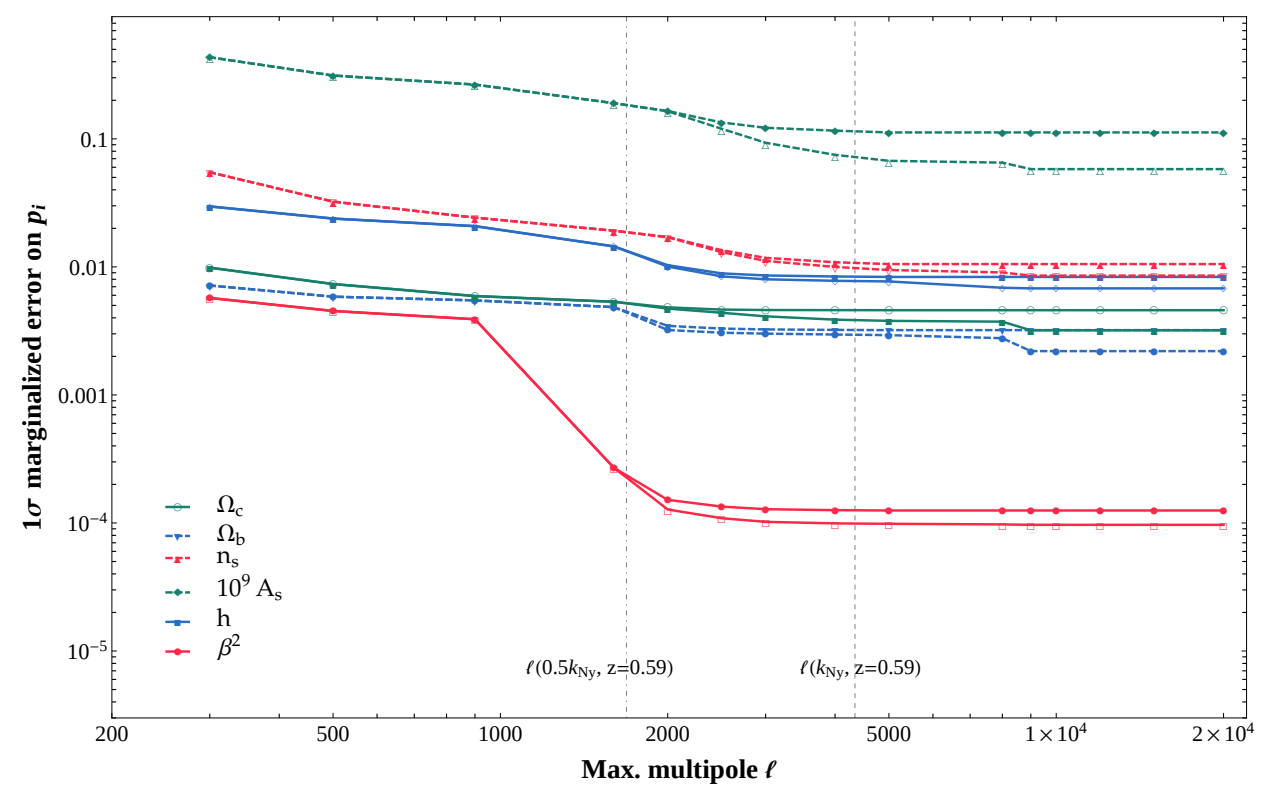

Figure 7. Variation in the 1- $\sigma$ fully marginalized errors for each cosmological parameter $p_{i}$ as a function of the maximum multipole used in the weak lensing Fisher forecast. Increasing the observed multipole range improves considerably the determination of a parameter, especially on the coupling $\beta^{2}$ (red solid line) and the initial amplitude of perturbations $10^{9} A_{s}$ (dashed green line). The double lines corresponding to each parameter, show how the error changes if a cut in the matter power spectrum $P(k)$ is performed at $k_{N y}$ (lower line) instead of a cut at $k_{N y} / 2$ (upper line, respectively). The vertical dashed grey lines mark the $\ell_{c u t}$ at the peak of the first redshift bins for the cases $\ell_{c u t, k_{N y} / 2}$ and $\ell_{c u t, k_{N y}}$.

in the case where non-linear information is used, increasing the maximum multipole $\ell_{\max }$ improves considerably the $1-\sigma$ error on the parameters, especially on the coupling $\beta^{2}$ and on the initial amplitude of scalar fluctuations $10^{9} A_{s}$. This is due to the fact that the extra signal on the coupling coming from the non-linear part of the power spectrum, the so called "bump", greatly enhances the constraints on the parameter estimation. The double lines corresponding to each parameter in figure 7, show how the error estimation is changed if scales up to $k_{N y}$ are included (lower line) compared to the upper line where only scales up to $k_{N y} / 2$ contribute. At small $\ell$ both lines are on top of each other and only start diverging at around $\ell=2000$, when the extra amount of information contained in highly non-linear scales starts becoming important. The most significant gains occur again on the parameters $\beta^{2}$ and $10^{9} A_{s}$.

\section{Combined results}

In figure 8 we show the 1-, 2- and 3- $\sigma$ confidence contours from the Fisher forecast for WL and GC. These confidence regions for each pair of parameters are obtained after marginalizing over all the other parameters. As it can be seen, some degeneracies are broken when combining the confidence ellipses from two different observations, for example in the case of the plane $\Omega_{b}, \beta^{2}$. Other parameter combinations, as $n_{s}, \beta^{2}$, show the same orientation of the ellipses for WL and GC, so that the combination of both probes does not help to disentangle the degeneracies. While GC constraints much better the usual parameters $\left\{h, 10^{9} \mathscr{A}_{s}, n_{s}, \Omega_{c}, \Omega_{b}\right\}$, WL constrains the coupling parameter $\beta^{2}$ much better which can be seen in the vertical orientation of the ellipses that correspond to $\beta^{2}$. Therefore combining the observations on GC and WL, as a future survey like Euclid will do, is a powerful way of constraining degenerate parameters in cosmology.

In table $\mathrm{X}$, we cite the 1- $\sigma$ fully-marginalised errors on the parameters $p_{i}$ for three different cases: a) using only linear CDE power spectra computed from our modified version of CAMB; b) applying a non-linear correction to these linear CDE power spectra using the latest version of Halofit from [3], which was designed for $\Lambda$ CDM-only; c) using the full coupled DE non-linear power spectra computed with our fitting functions following the procedure explained in section VIIC.

This shows the value of using the $\mathrm{N}$-body-calibrated fitting functions on the coupling $\beta^{2}$. Using the proper $\beta$-dependent nonlinear correction instead of the standard Halofit correction, the constraints on $\beta^{2}$ improve by more than an order of magnitude for WL and by a factor of order three for GC. This makes very clear the importance of applying non-linear corrections that depend on the parameter to be tested. 

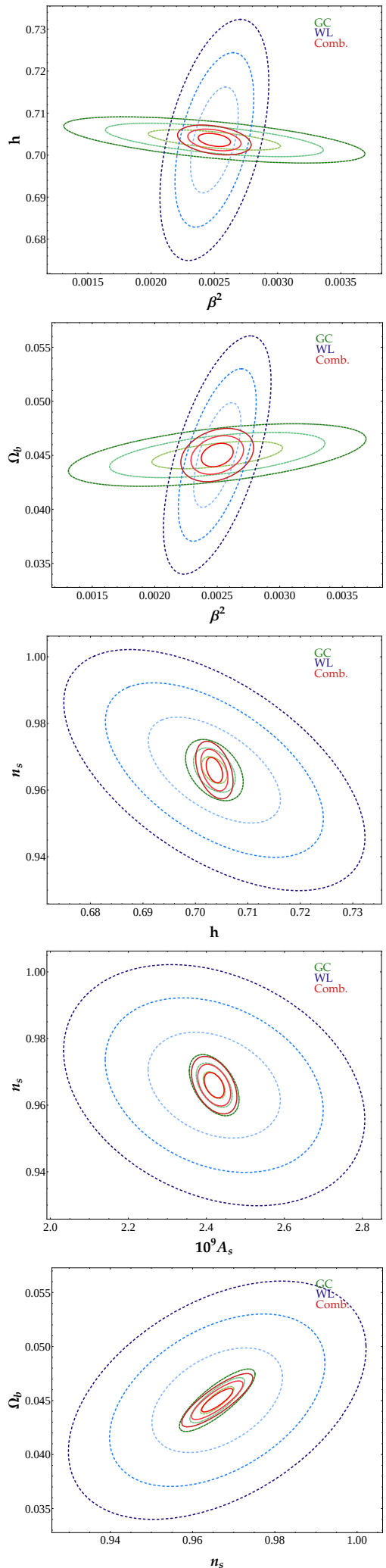
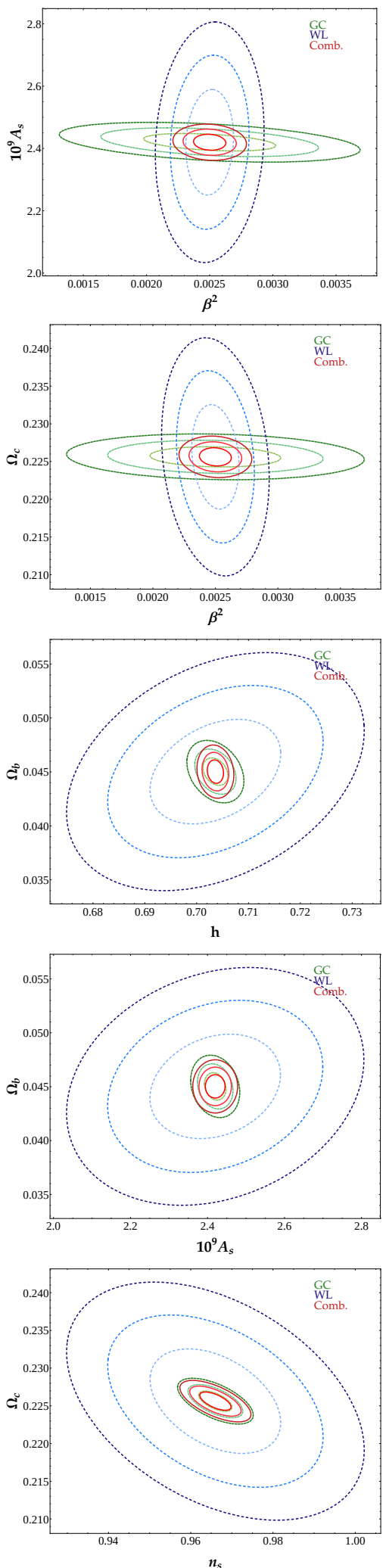
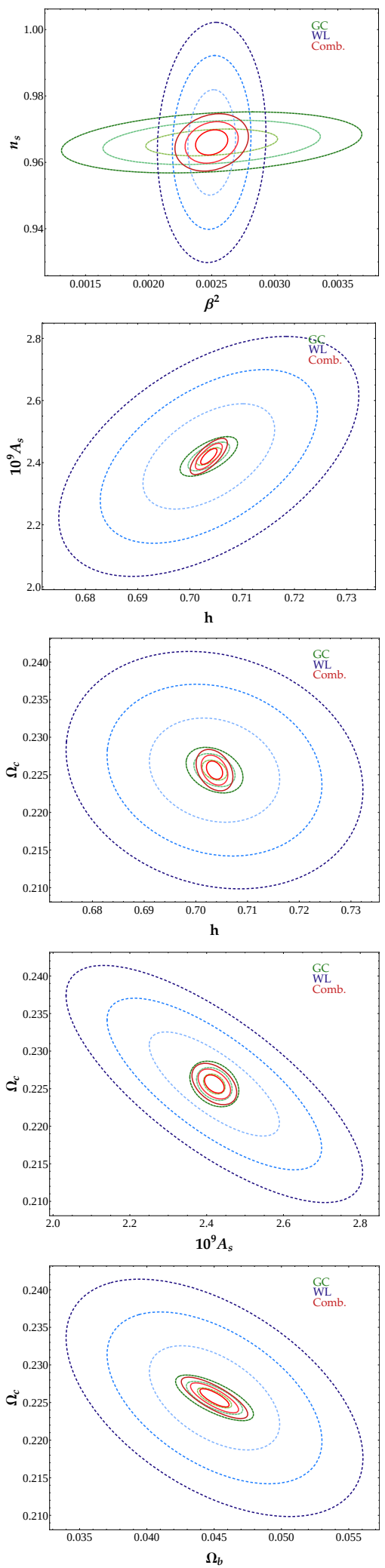

Figure 8. Marginalized confidence contour regions (1,2,3- $\sigma)$ for all cosmological parameters considered in this model. The blue dashed lines correspond to the WL Fisher forecast, while the green dashed lines correspond to the GC Fisher forecast both in our reference case. The red solid lines correspond to the combined Fisher matrix forecast. For combinations of the parameter $\beta^{2}$, WL and GC have similar figures of merit, but different orientations, while for other combinations of cosmological parameters, the estimation is dominated by the GC Fisher matrix estimation. 


\begin{tabular}{|c|c|c|c|c|c|c|}
\hline Parameter & $\beta^{2}$ & $h$ & $10^{9} \mathscr{A}_{S}$ & $n_{s}$ & $\Omega_{b}$ & $\Omega_{c}$ \\
\hline fiducial & 0.0025 & 0.7036 & 2.42 & 0.966 & 0.04503 & 0.2256 \\
\hline WL: 1- $\sigma$ abs. error, using: & & & & & & \\
\hline linear CDE & 0.0189 & 0.040 & 0.221 & 0.0139 & 0.0062 & 0.0127 \\
linear CDE+Halofit & 0.0184 & 0.044 & 0.256 & 0.0109 & 0.0066 & 0.0079 \\
non-linear CDE fitting functions & 0.000125 & 0.00835 & 0.112 & 0.0105 & 0.0032 & 0.0046 \\
\hline GC: 1- $\sigma$ abs. error, using: & & & & & & \\
\hline linear CDE & 0.0038 & 0.0097 & 0.117 & 0.0176 & 0.0021 & 0.0055 \\
linear CDE+Halofit & 0.0011 & 0.0029 & 0.024 & 0.0023 & 0.0007 & 0.0006 \\
non-linear CDE fitting functions & 0.00035 & 0.0016 & 0.018 & 0.0027 & 0.0008 & 0.0009 \\
comb. WL+GC: 1- $\sigma$ error, using: & & & & & & \\
\hline non-linear CDE fitting functions (abs.) & 0.000084 & 0.0010 & 0.0169 & 0.00251 & 0.00072 & 0.00080 \\
non-linear CDE fitting functions (rel.) & $3.4 \%$ & $0.16 \%$ & $0.7 \%$ & $0.26 \%$ & $1.6 \%$ & $0.35 \%$ \\
\hline
\end{tabular}

Table X. 1- $\sigma$ fully marginalized errors on the cosmological parameters for WL, GC and the combined Fisher matrix WL+GC, using three different power spectra. Linear CDE: Using only information from the linear power spectrum for the CDE model up to a scale of $k=$ $0.1 \mathrm{~h} / \mathrm{Mpc}$. Linear CDE+Halofit: Using the linear power spectrum for CDE plus a non-linear correction using the latest Halofit from [3]. Non-linear CDE fitting functions: Using the fully non-linear power spectra for CDE obtained from the fitting functions and the emulator as explained in $\mathrm{V}$, which we regard as the most reliable description of the model in this range of scales. In all these cases we are using our reference Fisher forecasts corresponding to the cutoff at $k_{N y} / 2$.

\section{CONCLUSIONS}

The goal of this paper is to exploit the cosmological information contained in the non-linear regime in order to improve parameter estimation from future large-scale observations. The main obstacle along this road is that we have accurate non-linear corrections for the matter power spectrum only for $\Lambda \mathrm{CDM}$ and a few other relatively simple variants, but not for the large variety of modifed gravity models that have been proposed in recent years.

The first part of this paper has then been devoted to the task of finding corrections to the linear power spectrum in the range of $k \approx 0.1-1 h / \mathrm{Mpc}$ for a selected class of modified gravity models, namely coupled dark energy. This model is indeed one of the simplest possible extensions of Einstein's gravity and depends entirely on a single parameter, the coupling constant $\beta$ (in addition to the standard ones). Employing the CoDECS suite of simulations [27] we build different fitting function models, such that when multiplied by the $\Lambda \mathrm{CDM}$ non-linear power spectrum (we use the estimator provided in ref. [6] ) they reproduce the $\mathrm{N}$-body results to an accuracy of $1 \%$, for scales $k$ between 0.1 and $5 \mathrm{~h} / \mathrm{Mpc}$ and a range in $z$, between 0 and 1.8. To achieve this accuracy in the fitting functions we need to perform a careful extraction and interpolation of the power spectrum from the simulation mesh.

The accurate fitting functions have been then employed to extend the regime of validity of the forecasts for future experiments. We focused on a Euclid-like survey that includes weak lensing and redshift-space distortions (galaxy clustering) and predicted the constraints in the cosmological parameters, with particular emphasis on the dark matter-dark energy coupling $\beta$. We find that $\beta$ is better constrained by weak lensing than by galaxy clustering (contrary to all the other standard parameters). We find that the extension into non-linear scales improves the constraints by more than an order of magnitude compared to previous results using only linear power spectra, but also by more than an order of magnitude in WL and a factor of three in GC compared to using a wrong $\Lambda \mathrm{CDM}$ Halofit non-linear correction. We also show that using the wrong non-linear power spectrum, can bias systematically the estimation of errors on the cosmological parameters, yielding systematic errors of the same order of magnitude as the statistical ones. This makes very clear that it is important to include the proper non-linear corrections to the parameter to be tested, especially for models beyond $\Lambda \mathrm{CDM}$ in which the small-scale gravitational dynamics are modified.

To make our forecast more realistic, we take into account all known sources of error entering the estimation of the power spectra and the fitting functions in the way of a reduced effective number density of galaxies and then perform a conservative cut of the power spectrum at half of the simulation Nyquist frequency, to avoid other sources of unknown numerical noise affecting the results. In the case of GC we include also a first approximation to the correction to redshift space distortions, caused by peculiar pairwise velocities at non-linear scales. We find that a space probe like Euclid will be able to constrain the coupling parameter $\beta^{2}$ around the fiducial value 0.0025 at $1-\sigma$ with a relative accuracy of $14 \%$ when using weak lensing alone, $5 \%$ when using only galaxy clustering and at $3.4 \%$ when combining both probes.

It is interesting to note that the most stringest constraint we obtain amounts to $\Delta \beta^{2} \approx 8 \cdot 10^{-5}$; this level of precision on the dark matter-dark energy coupling is not far from the current best limits reached with Solar System observations on a coupling to baryons [52], which can be translated in our notation as $\beta^{2} \leq 2 \cdot 10^{-5}$ at $1-\sigma$. 


\section{ACKNOWLEDGMENTS}

This research has been supported by DFG through the grant TRR33 “The Dark Universe". V.P. acknowledges support from the Heidelberg Graduate School for Fundamental Physics. S.C. acknowledges support from the Heidelberg Graduate School for Fundamental Physics and a previous support by the MICIT (Costa Rica). We thank E. Majerotto, D. Sapone, S. Camera for useful discussions.

[1] L. Casarini, G. La Vacca, L. Amendola, S. A. Bonometto, and A. V. Macciò. Non-linear weak lensing forecasts. JCAP, 3:026, March 2011. doi:10.1088/1475-7516/2011/03/026

[2] R. E. Smith, J. A. Peacock, A. Jenkins, S. D. M. White, C. S. Frenk, F. R. Pearce, P. A. Thomas, G. Efstathiou, and H. M. P. Couchman. Stable clustering, the halo model and non-linear cosmological power spectra. MNRAS, 341(4):1311-1332, June 2003. ISSN 0035-8711, 1365-2966. doi:10.1046/j.1365-8711.2003.06503.x URL http://mnras .oxfordjournals .org/content/341/4/1311.

[3] Ryuichi Takahashi, Masanori Sato, Takahiro Nishimichi, Atsushi Taruya, and Masamune Oguri. Revising the Halofit Model for the Nonlinear Matter Power Spectrum. arXiv:1208.2701, August 2012. URL http://arxiv.org/abs/1208.2701

[4] Katrin Heitmann, Martin White, Christian Wagner, Salman Habib, and David Higdon. The Coyote Universe. I. Precision determination of the nonlinear matter power spectrum. The Astrophysical Journal, 715(1):104, 2010. URL http://iopscience.iop.org/0004-637X/ $715 / 1 / 104$

[5] Earl Lawrence, Katrin Heitmann, Martin White, David Higdon, Christian Wagner, Salman Habib, and Brian Williams. The coyote universe. III. simulation suite and precision emulator for the nonlinear matter power spectrum. The Astrophysical Journal, 713(2):1322, 2010. URL http://iopscience.iop.org/0004-637X/713/2/1322

[6] Katrin Heitmann, Earl Lawrence, Juliana Kwan, Salman Habib, and David Higdon. The Coyote Universe Extended: Precision Emulation of the Matter Power Spectrum. The Astrophysical Journal, 780(1):111, 2014. URL http://iopscience.iop.org/0004-637X/780/ $1 / 111$

[7] Shankar Agarwal, Filipe B. Abdalla, Hume A. Feldman, Ofer Lahav, and Shaun A. Thomas. PkANN - I. Non-linear matter power spectrum interpolation through artificial neural networks. Monthly Notices of the Royal Astronomical Society, 424(2):1409-1418, August 2012. ISSN 00358711. doi:10.1111/j.1365-2966.2012.21326.x URL http://arxiv.org/abs/1203.1695. arXiv: 1203.1695.

[8] Shankar Agarwal, Filipe B. Abdalla, Hume A. Feldman, Ofer Lahav, and Shaun A. Thomas. PkANN - II. A non-linear matter power spectrum interpolator developed using artificial neural networks. Monthly Notices of the Royal Astronomical Society, 439(2):2102-2121, March 2014. ISSN 0035-8711, 1365-2966. doi:10.1093/mnras/stu090. URL http://arxiv.org/abs/1312.2101 arXiv: 1312.2101.

[9] Simeon Bird, Matteo Viel, and Martin G. Haehnelt. Massive Neutrinos and the Non-linear Matter Power Spectrum. arXiv:1109.4416, September 2011. doi:10.1111/j.1365-2966.2011.20222.x URL http://arxiv.org/abs/1109.4416 MNRAS, March 2012, Vol 420, Issue 3, 2551-2561.

[10] Benjamin Audren, Julien Lesgourgues, Simeon Bird, Martin G Haehnelt, and Matteo Viel. Neutrino masses and cosmological parameters from a Euclid-like survey: Markov Chain Monte Carlo forecasts including theoretical errors. Journal of Cosmology and Astroparticle Physics, 2013(01):026-026, January 2013. ISSN 1475-7516. doi:10.1088/1475-7516/2013/01/026. URL http://stacks.iop.org/ 1475-7516/2013/i=01/a=026?key=crossref .0594f c507c5faa23a2ba7c9ccb91b7e1.

[11] Jannis Bielefeld, Dragan Huterer, and Eric V. Linder. Cosmological Leverage from the Matter Power Spectrum in the Presence of Baryon and Nonlinear Effects. SciRate, November 2014. URL https://scirate.com/arxiv/1411.3725

[12] Irshad Mohammed and Uros Seljak. Analytic model for the matter power spectrum, its covariance matrix, and baryonic effects. Mon.Not.Roy.Astron.Soc., 445:3382-3400, December 2014. doi:10.1093/mnras/stu1972

[13] Martín Crocce and Román Scoccimarro. Renormalized cosmological perturbation theory. Phys. Rev. D, 73(6):063519, March 2006. doi:10.1103/PhysRevD.73.063519 URL http://link.aps.org/doi/10.1103/PhysRevD.73.063519

[14] Stefano Anselmi and Massimo Pietroni. Nonlinear power spectrum from resummed perturbation theory: a leap beyond the BAO scale. Journal of Cosmology and Astroparticle Physics, 2012(12):013, 2012. URL http://iopscience.iop.org/1475-7516/2012/12/ 013 .

[15] Massimo Pietroni. Flowing with Time: a New Approach to Nonlinear Cosmological Perturbations. JCAP, 0810:036, 2008. doi: 10.1088/1475-7516/2008/10/036.

[16] Daniel Baumann, Alberto Nicolis, Leonardo Senatore, and Matias Zaldarriaga. Cosmological Non-Linearities as an Effective Fluid. arXiv:1004.2488 [astro-ph, physics:gr-qc, physics:hep-th], April 2010. URL http://arxiv.org/abs/1004.2488 arXiv: 1004.2488.

[17] Christof Wetterich. The Cosmon model for an asymptotically vanishing time dependent cosmological 'constant'. Astron. Astrophys., 301:321-328, 1995.

[18] Luca Amendola. Coupled quintessence. Phys. Rev., D62:043511, 2000. doi:10.1103/PhysRevD.62.043511.

[19] Luca Amendola. Linear and non-linear perturbations in dark energy models. Phys. Rev., D69:103524, 2004. doi: 10.1103/PhysRevD.69.103524

[20] Valeria Pettorino and Carlo Baccigalupi. Coupled and Extended Quintessence: theoretical differences and structure formation. Phys. Rev., D77:103003, 2008. doi:10.1103/PhysRevD.77.103003

[21] Nico Wintergerst and Valeria Pettorino. Clarifying spherical collapse in coupled dark energy cosmologies. Phys. Rev., D82:103516, 2010. doi:10.1103/PhysRevD.82.103516.

[22] F. Saracco, M. Pietroni, N. Tetradis, V. Pettorino, and G. Robbers. Non-linear Matter Spectra in Coupled Quintessence. Phys. Rev., D82: 023528, 2010. doi:10.1103/PhysRevD.82.023528. 
[23] Marco Baldi, V. Pettorino, G. Robbers, and V. Springel. Hydrodynamical N-body simulations of coupled dark energy cosmologies. MNRAS, 403:1684-1702, April 2010. doi:10.1111/j.1365-2966.2009.15987.x

[24] Baojiu Li and John D. Barrow. N-Body Simulations for Coupled Scalar Field Cosmology. Phys. Rev., D83:024007, 2011. doi: 10.1103/PhysRevD.83.024007

[25] A.V. Maccio, C. Quercellini, R. Mainini, L. Amendola, and S.A. Bonometto. Coupled dark energy: Parameter constraints from N-body simulations. Phys. Rev. D, 69(12):123516, June 2004. doi:10.1103/PhysRevD.69.123516 URL http://link.aps.org/doi/10. 1103/PhysRevD.69.123516

[26] Edoardo Carlesi, Alexander Knebe, Geraint F. Lewis, Scott Wales, and Gustavo Yepes. Hydrodynamical simulations of coupled and uncoupled quintessence models I: Halo properties and the cosmic web. arXiv:1401.5005 [astro-ph], January 2014. URL http:// arxiv.org/abs/1401.5005 arXiv: 1401.5005.

[27] Marco Baldi. The codecs project: a publicly available suite of cosmological N-body simulations for interacting dark energy models. MNRAS, 422(2):1028-1044, May 2012. ISSN 0035-8711, 1365-2966. doi:10.1111/j.1365-2966.2012.20675.x. URL http://mnras . oxfordjournals.org/content/422/2/1028

[28] C. Penzo, A. V. Macciò, M. Baldi, L. Casarini, and J. Oñorbe. Effects of Coupled Dark Energy on the Milky Way and its Satellites. ArXiv e-prints, April 2015.

[29] Roberto Mainini and Silvio Bonometto. Mass functions in coupled Dark Energy models. arXiv:astro-ph/0605621, May 2006. doi: 10.1103/PhysRevD.74.043504 URL http://arxiv.org/abs/astro-ph/0605621. Phys.Rev.D74:043504,2006.

[30] Andrea V. Macciò, Aaron A. Dutton, and Frank C. van den Bosch. Concentration, spin and shape of dark matter haloes as a function of the cosmological model: WMAP 1, WMAP 3 and WMAP 5 results. Monthly Notices of the Royal Astronomical Society, 391(4):1940-1954, December 2008. ISSN 00358711, 13652966. doi:10.1111/j.1365-2966.2008.14029.x. URL http://mnras.oxfordjournals.org/ cgi/doi/10.1111/j.1365-2966.2008.14029.x

[31] Marco Baldi. Clarifying the effects of interacting dark energy on linear and non-linear structure formation processes. MNRAS, 414(1): 116-128, June 2011. ISSN 0035-8711, 1365-2966. doi:10.1111/j.1365-2966.2011.18263.x URL http://mnras.oxfordjournals. org/content/414/1/116

[32] Weiguang Cui, Marco Baldi, and Stefano Borgani. The halo mass function in interacting Dark Energy models. arXiv:1201.3568, January 2012. URL http://arxiv.org/abs/1201.3568

[33] Marco Baldi, Jounghun Lee, and Andrea V. Maccio. The effect of coupled dark energy on the alignment between dark matter and galaxy distributions in clusters. The Astrophysical Journal, 732(2):112, 2011. URL http://iopscience .iop.org/0004-637X/732/2/112.

[34] P. M. Sutter, Edoardo Carlesi, Benjamin D. Wandelt, and Alexander Knebe. On the observability of coupled dark energy with cosmic voids. arXiv:1406.0511 [astro-ph], June 2014. URL http://arxiv.org/abs/1406.0511.

[35] Marco Baldi. Dark Energy Simulations. Phys.Dark Univ., 1:162-193, 2012. doi:10.1016/j.dark.2012.10.004

[36] Valeria Pettorino, Luca Amendola, Carlo Baccigalupi, and Claudia Quercellini. Constraints on coupled dark energy using CMB data from WMAP and SPT. arXiv:1207.3293, July 2012. URL http://arxiv.org/abs/1207.3293.

[37] Valeria Pettorino. Testing modified gravity with Planck: the case of coupled dark energy. Phys. Rev. D 88,, $063519,2013$.

[38] Luca Amendola and Claudia Quercellini. Skewness as a test of the equivalence principle. Phys. Rev. Lett., 92:181102, 2004. doi: 10.1103/PhysRevLett.92.181102.

[39] Jun-Qing Xia. Constraint on coupled dark energy models from observations. Phys. Rev., D80:103514, $2009 . \quad$ doi: 10.1103/PhysRevD.80.103514

[40] Planck Collaboration. Planck 2015 results. XIV. Dark energy and modified gravity. arXiv:1502.01590 [astro-ph], February 2015. URL http://arxiv.org/abs/1502.01590 arXiv: 1502.01590.

[41] Paolo Creminelli, Jérôme Gleyzes, Lam Hui, Marko Simonović, and Filippo Vernizzi. Single-Field Consistency Relations of Large Scale Structure. Part III: Test of the Equivalence Principle. Journal of Cosmology and Astroparticle Physics, 2014(06):009-009, June 2014. ISSN 1475-7516. doi:10.1088/1475-7516/2014/06/009 URL http://arxiv.org/abs/1312.6074 arXiv: 1312.6074.

[42] Sophie C. F. Morris and Anne M. Green. Cosmic microwave background constraints on coupled dark matter. Phys.Lett., B741:51-54, December 2014. doi:10.1016/j.physletb.2014.12.025

[43] Paul Hamilton, Matt Jaffe, Philipp Haslinger, Quinn Simmons, Holger Müller, and Justin Khoury. Atom-interferometry constraints on dark energy. 2015.

[44] Luca Amendola, Valeria Pettorino, Claudia Quercellini, and Adrian Vollmer. Testing coupled dark energy with next-generation largescale observations. Physical Review D, 85(10), May 2012. ISSN 1550-7998, 1550-2368. doi:10.1103/PhysRevD.85.103008 URL http://link.aps.org/doi/10.1103/PhysRevD.85.103008.

[45] F. Saracco, M. Pietroni, N. Tetradis, V. Pettorino, and G. Robbers. Non-linear Matter Spectra in Coupled Quintessence. Phys. Rev., D82: 023528, 2010. doi:10.1103/PhysRevD.82.023528.

[46] Julien Lesgourgues, Sabino Matarrese, Massimo Pietroni, and Antonio Riotto. Non-linear power spectrum including massive neutrinos: the time-RG flow approach. Journal of Cosmology and Astroparticle Physics, 2009(06):017, 2009. URL http://iopscience.iop. org/1475-7516/2009/06/017

[47] R. Laureijs et al. Euclid Definition Study Report. arXiv:1110.3193 [astro-ph], October 2011. URL http://arxiv.org/abs/1110. 3193 arXiv: 1110.3193.

[48] Luca Amendola et al. Cosmology and fundamental physics with the Euclid satellite. arXiv:1206.1225 [astro-ph, physics:gr-qc, physics:hep-ph], June 2012. URL http://arxiv.org/abs/1206.1225 arXiv: 1206.1225.

[49] F. Lucchin and S. Matarrese. Power Law Inflation. Phys. Rev., D32:1316, 1985. doi:10.1103/PhysRevD.32.1316

[50] C. Wetterich. Cosmology and the Fate of Dilatation Symmetry. Nucl. Phys., B302:668, 1988. doi:10.1016/0550-3213(88)90193-9.

[51] Marco Baldi. Time-dependent couplings in the dark sector: from background evolution to non-linear structure formation. MNRAS, 411: 1077-1103, February 2011. doi:10.1111/j.1365-2966.2010.17758.x

[52] K. A. Olive et al. Review of Particle Physics. Chin. Phys., C38:090001, 2014. doi:10.1088/1674-1137/38/9/090001. 
[53] Clifford M. Will. The Confrontation between general relativity and experiment. Living Rev.Rel., 9:3, 2005.

[54] B. Bertotti, L. Iess, and P. Tortora. A test of general relativity using radio links with the Cassini spacecraft. Nature, 425:374, 2003. doi:10.1038/nature01997.

[55] P. Hamilton, M. Jaffe, P. Haslinger, Q. Simmons, H. Müller, and J. Khoury. Atom-interferometry constraints on dark energy. ArXiv e-prints, February 2015.

[56] F. Pace, J. C. Waizmann, and M. Bartelmann. Spherical collapse model in dark energy cosmologies. arXiv:1005.0233, 2010.

[57] Andrea V. Macciò, Claudia Quercellini, Roberto Mainini, Luca Amendola, and Silvio A. Bonometto. N-body simulations for coupled dark energy: halo mass function and density profiles. Phys. Rev., D69:123516, 2004. doi:10.1103/PhysRevD.69.123516

[58] E. Carlesi, A. Knebe, G. F. Lewis, S. Wales, and G. Yepes. Hydrodynamical simulations of coupled and uncoupled quintessence models - I. Halo properties and the cosmic web. MNRAS, 439:2943-2957, April 2014. doi:10.1093/mnras/stu150.

[59] Volker Springel. The cosmological simulation code GADGET-2. Mon. Not. Roy. Astron. Soc., 364:1105-1134, 2005.

[60] E. Komatsu, K. M. Smith, J. Dunkley, C. L. Bennett, B. Gold, G. Hinshaw, N. Jarosik, D. Larson, M. R. Nolta, L. Page, D. N. Spergel, M. Halpern, R. S. Hill, A. Kogut, M. Limon, S. S. Meyer, N. Odegard, G. S. Tucker, J. L. Weiland, E. Wollack, and E. L. Wright. Seven-Year Wilkinson Microwave Anisotropy Probe (WMAP) Observations: Cosmological Interpretation. arXiv:1001.4538, January 2010. doi:10.1088/0067-0049/192/2/18. URL http://arxiv.org/abs/1001.4538. Astrophys.J.Suppl.192:18,2011.

[61] P.A.R. Ade et al. Planck 2015 results. XIII. Cosmological parameters. 2015.

[62] Weiguang Cui, Lei Liu, Xiaohu Yang, Yu Wang, Longlong Feng, and Volker Springel. An ideal mass assignment scheme for measuring the Power Spectrum with FFTs. arXiv preprint arXiv:0804.0070, 2008. URL http://arxiv.org/abs/0804.0070.

[63] Stéphane Colombi, Andrew Jaffe, Dmitri Novikov, and Christophe Pichon. Accurate estimators of power spectra in $N$-body simulations. Monthly Notices of the Royal Astronomical Society, 393(2):511-526, February 2009. ISSN 00358711, 13652966. doi:10.1111/j.13652966.2008.14176.x URL http://mnras.oxfordjournals.org/cgi/doi/10.1111/j.1365-2966.2008.14176.x

[64] Adrian Jenkins, C. S. Frenk, F. R. Pearce, P. A. Thomas, J. M. Colberg, S. D. M. White, H. M. P. Couchman, J. A. Peacock, G. Efstathiou, and A. H. Nelson. Galaxy clustering determined from numerical cosmological simulations. arXiv:astro-ph/9906039, June 1999. URL http://arxiv.org/abs/astro-ph/9906039

[65] Eric W. Weisstein. Least Squares Fitting, . URL http://mathworld.wolfram.com/LeastSquaresFitting.html

[66] Eric W. Weisstein. Correlation Coefficient, . URL http://mathworld.wolfram.com/CorrelationCoefficient.html

[67] Martín Crocce and Román Scoccimarro. Memory of initial conditions in gravitational clustering. Physical Review D, 73(6), March 2006. ISSN 1550-7998, 1550-2368. doi:10.1103/PhysRevD.73.063520. URL http://link. aps.org/doi/10.1103/PhysRevD.73. 063520 .

[68] Massimo Pietroni, Gianpiero Mangano, Ninetta Saviano, and Matteo Viel. Coarse-Grained Cosmological Perturbation Theory. arXiv:1108.5203 [astro-ph, physics:hep-th], August 2011. URL http://arxiv.org/abs/1108.5203. arXiv: 1108.5203.

[69] Ryuichi Takahashi, Naoki Yoshida, Masahiro Takada, Takahiko Matsubara, Naoshi Sugiyama, Issha Kayo, Takahiro Nishimichi, Shun Saito, and Atsushi Taruya. Non-Gaussian Error Contribution to Likelihood Analysis of the Matter Power Spectrum. The Astrophysical Journal, 726(1):7, January 2011. ISSN 0004-637X, 1538-4357. doi:10.1088/0004-637X/726/1/7. URL http://arxiv.org/abs/ 0912.1381, arXiv: 0912.1381.

[70] Daniel Baumann, Alberto Nicolis, Leonardo Senatore, and Matias Zaldarriaga. Cosmological non-linearities as an effective fluid. Journal of Cosmology and Astroparticle Physics, 2012(07):051, 2012. URL http://iopscience.iop.org/1475-7516/2012/07/051.

[71] Alessandro Manzotti, Marco Peloso, Massimo Pietroni, Matteo Viel, and Francisco Villaescusa-Navarro. A coarse grained perturbation theory for the Large Scale Structure, with cosmology and time independence in the UV. arXiv:1407.1342 [astro-ph, physics:gr-qc, physics:hep-th], July 2014. URL/http://arxiv.org/abs/1407.1342 arXiv: 1407.1342.

[72] Antony Lewis, Anthony Challinor, and Anthony Lasenby. Efficient Computation of CMB anisotropies in closed FRW models. arXiv:astro-ph/9911177, November 1999. doi:10.1086/309179 URL http://arxiv.org/abs/astro-ph/9911177 Astrophys.J.538:473-476,2000.

[73] P. Fosalba, M. Crocce, E. Gaztanaga, and F. J. Castander. The MICE Grand Challenge Lightcone Simulation I: Dark matter clustering. arXiv preprint arXiv:1312.1707, 2013. URL http://arxiv.org/abs/1312.1707.

[74] Hume A. Feldman, Nick Kaiser, and John A. Peacock. Power Spectrum Analysis of Three-Dimensional Redshift Surveys. The Astrophysical Journal, 426:23, May 1994. ISSN 0004-637X, 1538-4357. doi:10.1086/174036 URL http://arxiv.org/abs/astro-ph/ 9304022 arXiv: astro-ph/9304022.

[75] Max Tegmark, Andrew Hamilton, Michael Strauss, Michael Vogeley, and Alexander Szalay. Measuring the galaxy power spectrum with future redshift surveys. The Astrophysical Journal, 499(2):555-576, June 1998. ISSN 0004-637X, 1538-4357. doi:10.1086/305663 URL http://arxiv.org/abs/astro-ph/9708020 arXiv: astro-ph/9708020.

[76] Hee-Jong Seo and Daniel J. Eisenstein. Improved forecasts for the baryon acoustic oscillations and cosmological distance scale. The Astrophysical Journal, 665(1):14-24, August 2007. ISSN 0004-637X, 1538-4357. doi:10.1086/519549. URL http://arxiv.org/ abs/astro-ph/0701079 arXiv: astro-ph/0701079.

[77] Hee-Jong Seo and Daniel J. Eisenstein. Baryonic acoustic oscillations in simulated galaxy redshift surveys. The Astrophysical Journal, 633(2):575-588, November 2005. ISSN 0004-637X, 1538-4357. doi:10.1086/491599 URL http://arxiv.org/abs/astro-ph/ 0507338 arXiv: astro-ph/0507338.

[78] Elena Sellentin, Miguel Quartin, and Luca Amendola. Breaking the spell of Gaussianity: forecasting with higher order Fisher matrices. Monthly Notices of the Royal Astronomical Society, 441(2):1831-1840, May 2014. ISSN 0035-8711, 1365-2966. doi: 10.1093/mnras/stu689 URL http://arxiv.org/abs/1401.6892 arXiv: 1401.6892.

[79] Charles Alcock and Bohdan Paczyński. An evolution free test for non-zero cosmological constant. Nature, 281(5730):358-359, October 1979. doi:10.1038/281358a0 URL/http://www.nature.com/nature/journal/v281/n5730/abs/281358a0.html

[80] A. N. Taylor, T. D. Kitching, D. J. Bacon, and A. F. Heavens. Probing dark energy with the shear-ratio geometric test. Monthly Notices of the Royal Astronomical Society, 374(4):1377-1403, February 2007. ISSN 0035-8711, 1365-2966. doi:10.1111/j.1365- 
2966.2006.11257.x URL http://mnras.oxfordjournals.org/content/374/4/1377

[81] Hee-Jong Seo, Ethan R. Siegel, Daniel J. Eisenstein, and Martin White. Nonlinear structure formation and the acoustic scale. The Astrophysical Journal, 686(1):13, 2008. URL http://iopscience.iop.org/0004-637X/686/1/13

[82] Luca Amendola, Valeria Pettorino, Claudia Quercellini, and Adrian Vollmer. Testing coupled dark energy with next-generation largescale observations. Physical Review D, 85(10), May 2012. ISSN 1550-7998, 1550-2368. doi:10.1103/PhysRevD.85.103008 URL http://link.aps.org/doi/10.1103/PhysRevD.85.103008.

[83] P. Fosalba, M. Crocce, E. Gaztanaga, and F. J. Castander. The MICE grand challenge lightcone simulation i: Dark matter clustering. arXiv preprint arXiv:1312.1707, 2013. URL http://arxiv.org/abs/1312.1707.

[84] Nick Kaiser. Clustering in real space and in redshift space. Monthly Notices of the Royal Astronomical Society, 227:1-21, July 1987. ISSN 0035-8711. URL http://adsabs . harvard . edu/abs/1987MNRAS . 227 . . .1K

[85] Sylvain de la Torre and Luigi Guzzo. Modelling non-linear redshift-space distortions in the galaxy clustering pattern: systematic errors on the growth rate parameter. arXiv:1202.5559 [astro-ph], February 2012. URL http://arxiv.org/abs/1202.5559. arXiv: 1202.5559.

[86] Román Scoccimarro. Redshift-space distortions, pairwise velocities, and nonlinearities. Phys. Rev. D, 70(8):083007, October 2004. doi:10.1103/PhysRevD.70.083007. URL http://link.aps.org/doi/10.1103/PhysRevD.70.083007

[87] Atsushi Taruya, Takahiro Nishimichi, and Shun Saito. Baryon acoustic oscillations in 2d: Modeling redshift-space power spectrum from perturbation theory. Phys. Rev. D, 82(6):063522, September 2010. doi:10.1103/PhysRevD.82.063522. URL http://link.aps.org/ doi/10.1103/PhysRevD.82.063522

[88] Yun Wang, Chia-Hsun Chuang, and Christopher M. Hirata. Toward More Realistic Forecasting of Dark Energy Constraints from Galaxy Redshift Surveys. arXiv:1211.0532 [astro-ph], November 2012. URL http://arxiv.org/abs/1211.0532. arXiv: 1211.0532.

[89] Alvaro Orsi, C. M. Baugh, C. G. Lacey, A. Cimatti, Y. Wang, and G. Zamorani. Probing dark energy with future redshift surveys: A comparison of emission line and broad band selection in the near infrared. Mon.Not.Roy.Astron.Soc., 405:1006, 2010. doi:10.1111/j.13652966.2010.16585.x 


\section{Appendix A: Parameters of the fitting models M2 and M7}

Model M2 and M7 have each 5 coefficients, which are third order polynomial function of $\beta$ and $z$ :

$$
\begin{aligned}
a_{i}(\beta, z) & =q_{i 1}+q_{i 2} \beta+q_{i 3} z+q_{i 4} \beta z \\
& +q_{i 5} \beta^{2}+q_{i 6} z^{2}+q_{i 7} z^{3}+q_{i 8} \beta^{3}
\end{aligned}
$$

Since we have 5 coefficients and 8 free parameters for each coefficient, we have a total of 40 free parameters. They can be represented in a matrix $q_{i j}$ by: $A_{i}=q_{i j} B_{j}$, where the vector $A_{i}=a_{i}(\beta, z)$ is the vector of coefficients and $B_{j}=$ $\left(1, \beta, z, \beta z, \beta^{2}, z^{2}, \beta^{3}, z^{3}\right)$ the vector of independent variables and the indices are defined in the ranges: $i=1, \ldots, 5, j=1, \ldots, 8$. The corresponding matrix $q_{i j}$ for model M7 is:

$$
q_{i j}(M 7)=\left(\begin{array}{cccccccc}
0.00689754 & -0.200001 & -0.0112943 & 0.157222 & -1.40171 & 0.0129681 & -3.99345 & -0.00629434 \\
-0.0706221 & 1.45187 & 0.236402 & -1.26467 & 9.13266 & -0.300832 & 9.81754 & 0.120649 \\
0.069102 & -1.44396 & -0.24268 & 1.26135 & -9.23052 & 0.311995 & -12.1568 & -0.12561 \\
1.19797 & 0.632936 & -1.74767 & 0.920634 & -8.47056 & 1.46434 & 42.7015 & -0.471948 \\
0.684838 & 1.15722 & -2.15405 & 3.93688 & -5.29011 & 3.42683 & -43.9295 & -1.18691
\end{array}\right)
$$

and the coefficient matrix for model M2 is:

$$
q_{i j}(M 2)=\left(\begin{array}{cccccccc}
0.00301472 & -0.0961879 & -0.00897697 & 0.0389941 & -0.860642 & 0.0131329 & -4.73786 & -0.00552858 \\
-0.028584 & 0.615003 & 0.111671 & -0.371008 & 4.94379 & -0.138279 & 20.3258 & 0.0512362 \\
0.0132728 & -0.345125 & -0.0701132 & 0.115389 & -3.11777 & 0.102871 & -16.9949 & -0.0408223 \\
2.91757 & 1.03545 & -5.16595 & 1.69976 & -22.1649 & 4.33453 & 120.513 & -1.35074 \\
0.867566 & 2.92363 & -1.14174 & 3.4356 & -2.96193 & 1.91413 & -113.348 & -0.684269
\end{array}\right)
$$

Applying a singular value decomposition on both matrices, shows that they have a very similar structure, therefore since the sigmoidal functions M2 and M7 have a related functional form, the matrices describe roughly the same information. 